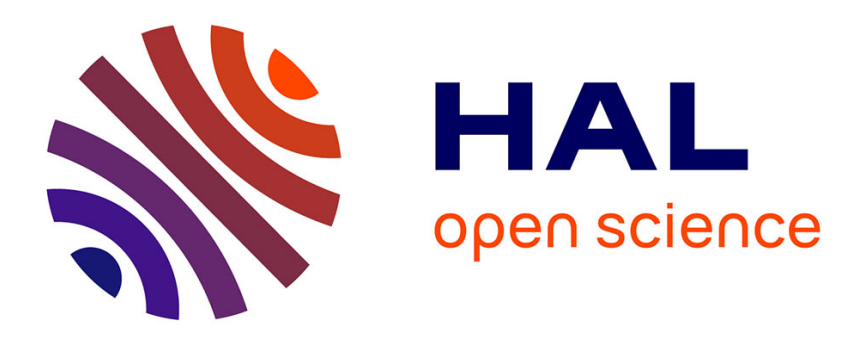

\title{
Impacts de la météorologie sur la demande de trafic
}

\author{
Nour Eddin El Faouzi, Jérémie Buton, Romain Billot
}

\section{To cite this version:}

Nour Eddin El Faouzi, Jérémie Buton, Romain Billot. Impacts de la météorologie sur la demande de trafic. RTS - Recherche Transports Sécurité, 2013, 2013 (01), pp.21-46. 10.4074/S0761898013001039 . hal-00921636

\section{HAL Id: hal-00921636 https://hal.science/hal-00921636}

Submitted on 8 Feb 2018

HAL is a multi-disciplinary open access archive for the deposit and dissemination of scientific research documents, whether they are published or not. The documents may come from teaching and research institutions in France or abroad, or from public or private research centers.
L'archive ouverte pluridisciplinaire HAL, est destinée au dépôt et à la diffusion de documents scientifiques de niveau recherche, publiés ou non, émanant des établissements d'enseignement et de recherche français ou étrangers, des laboratoires publics ou privés. 


\title{
Impacts de la météorologie sur la demande de trafic
}

\section{Effects of adverse weather conditions on traffic demand}

\author{
Nour-Eddin El Faouzi · Jérémie Buton · Romain Billot
}

Reçu le 8 juin 2012 ; accepté le 20 décembre 2012

(c) IFSTTAR et Éditions NecPlus 2013

Résumé L'objectif de cet article est de mettre en évidence les effets de la météorologie sur la demande de trafic. Une étude approfondie de la littérature est d'abord réalisée : d'une part une analyse des conséquences sur la demande de déplacement au sens large à travers le prisme du modèle à quatre étapes (à partir d'enquêtes principalement) et d'autre part un focus sur la demande de trafic, en synthétisant les analyses réalisées à partir des données boucles. L'analyse montre ainsi que certains événements météorologiques, comme la pluie et surtout la neige, conduisent à des modifications dans les comportements (transfert modal, annulation du déplacement. ..) et à une baisse de la demande de trafic. La nature et l'amplitude des changements opérés dépendent de nombreux facteurs, comme la période du jour (journée/nuit), de la semaine (semaine/week-end), du milieu (urbain/interurbain), du motif du déplacement

Remerciements Ces travaux ont été en partie réalisés dans le cadre de l'action COST TU0702 Real-time Monitoring, Surveillance and Control of Road Networks under Adverse Weather Conditions (www.cost-TU0702.org) [35] et de l'action Marie-Curie Optimum (Optimised ITS-based Tools for Intelligent Urban Mobility, www.optimum-project.net).

Nour-Eddin El Faouzi $(\triangle)$

Université de Lyon, F-69000 Lyon, France

IFSTTAR, COSYS/LICIT, F-69675, Bron

ENTPE, LICIT, F-69518, Vaulx-en-Velin

25 av. François Mitterrand, Cité des mobilités,

Case 24, F-69675 Bron cedex

e-mail : nour-eddin.elfaouzi@ifsttar.fr

Jérémie Buton $(\bowtie)$

DRIEA/DIRIF/DET, 79C avenue du Maréchal de Lattre de Tassigny, F-94100 Créteil cedex, France

e-mail : jeremie.buton@developpement-durable.gouv.fr

Romain Billot $(\square)$

Université de Lyon, F-69000 Lyon, France

IFSTTAR, COSYS/LICIT, F-69675, Bron

ENTPE, LICIT, F-69518, Vaulx-en-Velin

25 av. François Mitterrand, Cité des mobilités,

Case 24, F-69675 Bron cedex

e-mail : romain.billot@ifsttar.fr (contraint/non contraint)... Cette bibliographie est ensuite complétée par des analyses statistiques et des modélisations sur des données opérationnelles recueillies en milieu interurbain (section Lyon-Chambéry de l'A43) et sur la route $\mathrm{n}^{\circ} 3$ des autoroutes urbaines de Tokyo.

Mots clés demande de trafic - météorologie défavorable . neige pluie

Abstract The goal of this study is to highlight the effects of adverse weather conditions on traffic demand. The literature review underlines that some events, especially rain and snow, induce significant changes on both travel demand and traffic operations. These changes have several origins (modal shift, time departure postponement, travel cancellation...) and depend on many factors, such as time of day (day/night), week (weekdays/weekend), environment (urban/interurban area), trip purpose (discretionary/nondiscretionary)... The literature review is therefore divided in two parts: a study of surveys that reveals the impact of weather on travel choices, and then statistical analysis conducted on traffic data collected from loop detectors. This preliminary work is completed by statistical analysis and modeling on operational interurban data (a section of the A43 French Highway) and urban data (route $n^{\circ} 3$, Tokyo).

Keywords traffic demand adverse weather conditions . snow $\cdot$ rain

\section{Introduction}

Les enjeux liés à la maîtrise des diverses composantes du trafic et de la demande en particulier sont devenus particulièrement cruciaux. Il est nécessaire d'optimiser l'exploitation des infrastructures de transport, et cela ne peut se faire qu'en collant au mieux à la demande. Pouvoir la réguler et agir sur celle-ci représente donc un enjeu majeur dans une optique d'une meilleure optimisation du système de transport. 
La compréhension du fonctionnement des infrastructures de transport et de leur gestion passe donc avant tout par une parfaite appréhension des différents facteurs qui influencent les déplacements. Les conditions météorologiques, de par leur impact significatif sur le trafic, peuvent être vues comme une source importante d'incertitude dégradant le fonctionnement nominal des réseaux de transport. Plusieurs études ont mis en évidence les effets notables de certains événements météorologiques sur la mobilité, sur la sécurité, mais aussi sur le comportement des usagers. Les performances des infrastructures de transport en cas de conditions météorologiques dégradées ont fait l'objet de nombreuses recherches, notamment concernant la vitesse libre et la capacité de l'infrastructure. Prevedouros et Kongsil ont par exemple synthétisé un ensemble d'études traitant de ces deux derniers points et concluent à une réduction moyenne des vitesses de $7,6 \mathrm{~km} / \mathrm{h}$ en cas de pluie fine, et de $31,6 \mathrm{~km} / \mathrm{h}$ en cas de fortes pluies. La chute de capacité moyenne atteindrait quant à elle les $8,4 \%$ lors d'une pluie fine, et $20 \%$ lors d'une pluie forte. L'étude de l'influence de la météorologie sur la demande reste pour sa part jusqu'à aujourd'hui très peu explorée [1].

C'est dans le cadre d'un partenariat avec les réseaux d'autoroutes Area (Société des autoroutes Rhône-Alpes) que notre étude tient son origine. Le projet TPTEO vise à intégrer les effets de la météorologie dans les stratégies de gestion du trafic et d'information des usagers. À ce titre, un outil de prévision à court terme des temps de parcours sur le réseau autoroutier interurbain d'Area est élaboré par le Licit (Laboratoire ingénierie circulation transports, unité mixte de recherche de l'Ifsttar et de l'ENTPE). L'intégration des conséquences de la météorologie sur la demande permettrait l'amélioration de l'efficacité du moteur de prévisions [2].

Il s'agira donc, dans la suite, de quantifier les effets de la météorologie défavorable sur la demande. On rappelle que la demande se définit par la quantité de trafic qui cherche à s'écouler sur une infrastructure. On parle, selon les cas, soit de la demande de déplacement, soit de la demande de trafic pour une infrastructure donnée. La demande de déplacement correspond au nombre total de déplacements qui vont chercher à être réalisés au travers d'un ensemble de modes disponibles sur un réseau défini, alors que la demande de trafic sur une infrastructure donnée n'est mesurée (par le débit) que lorsque le trafic est fluide. En cas de congestion sur le réseau, il convient de noter que l'on mesure l'offre et non la demande.

Ces effets, s'ils existent bel et bien, sont-ils assez importants pour être pris en compte d'un point de vue opérationnel ? Il est également nécessaire de s'interroger sur les facteurs qui conduisent à observer des variations dans la demande. Quels sont les facteurs qui expliquent le plus ces variations? Dans quelles proportions? De quels paramètres dépendent-ils ? L'étude de ces facteurs permettrait de mieux appréhender les variations qui peuvent subvenir en cas de conditions météorologiques dégradées notamment, et ainsi d'améliorer la gestion actuelle des infrastructures.

Les deux parties suivantes de l'article permettent de dresser l'état de l'art des études réalisées dans le domaine. Dans la première de ces parties, nous tentons donc de décrire quels peuvent être les impacts d'une météorologie défavorable sur la demande à travers le prisme du modèle à quatre étapes. Un certain nombre d'enquêtes, d'expérience ou de plans d'expérience sont examinés à cette occasion. La seconde partie se concentre sur l'examen des variations des volumes de déplacement rapportées dans la littérature dans le cas de conditions météorologiques dégradées. Ce sont ainsi principalement des études se basant sur des données de trafic issues de boucles de comptage qui sont synthétisées. Les effets de la neige et de la pluie, facteurs ayant a priori le plus d'influence, sont particulièrement approfondis. La troisième partie permet ensuite de s'intéresser de près à un cas interurbain (la section Lyon-Chambéry de l'autoroute $\mathrm{A} 43$ ) et à un cas urbain (la route $\mathrm{n}^{\circ} 3$ du réseau des voies rapides urbaines de la ville de de Tokyo). Les données des boucles de comptage sont découpées en échantillons comparables puis analysées par diverses méthodes statistiques (comparaison des intervalles de confiance des moyennes d'échantillon sec, pluvieux et neigeux, diagrammes en boîte, tests de Mann-Whitney...). Enfin, nous quantifions dans la dernière partie les variations de débit qui peuvent se produire sous certains facteurs météorologiques (neige, pluie, température...) en construisant différents modèles de prévisions de trafic.

\section{Météorologie et choix de déplacement}

\section{Comment étudier ces impacts ?}

L'objet de cette première partie est de dresser un état des lieux le plus complet possible de la littérature existante sur l'impact que peuvent avoir des conditions météorologiques sur la demande de trafic. De nombreux auteurs s'intéressent depuis plusieurs années déjà aux liens qui existent entre météorologie et trafic, dans la perspective notamment d'en identifier les impacts sur les variables microscopiques de trafic, comme la vitesse libre et la capacité de l'infrastructure. L'autre domaine de recherche concerne essentiellement l'influence d'une météorologie défavorable sur l'accidentologie. L'analyse des effets de la métérologie sur la demande a fait parfois l'objet d'investigations indirectes. Citons par exemple Datla et Sharma, qui notent l'existence d'une interaction forte entre météorologie, accidentologie et volume de trafic. L'impact 


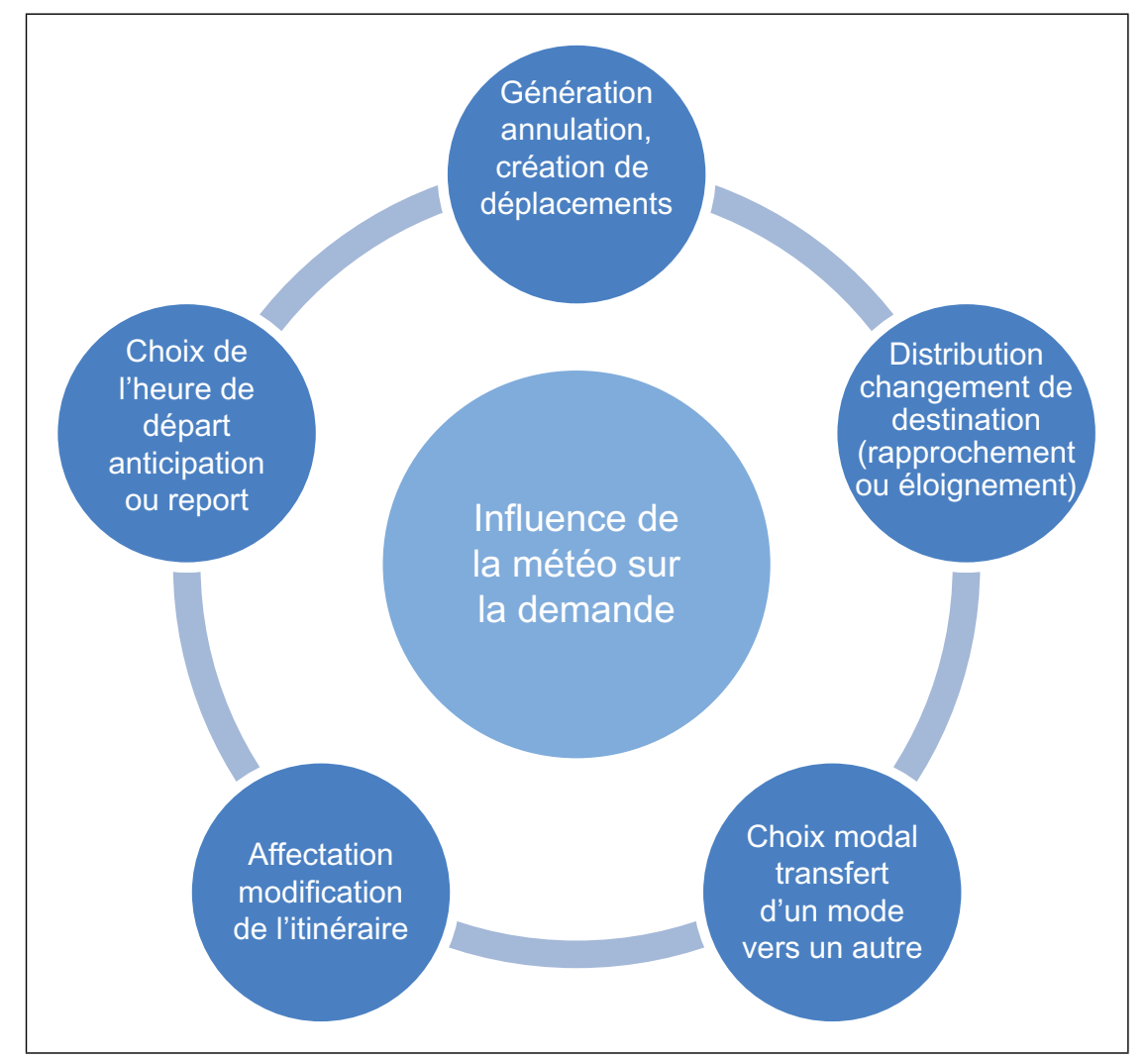

Fig. 1 Les modifications possibles des choix de déplacement d'un individu en cas de conditions météo dégradées

sur la demande est également souvent étudié sous l'angle du changement climatique [2] [3] [4] [5] [6].

Les effets de la météorologie sur les comportements des usagers et in fine sur la structure de la demande sont multiples (voir Figure 1). De nombreuses études ont, par exemple, mis en évidence l'impact d'une météorologie défavorable sur les vitesses, les distances inter-véhiculaires, etc. Nous allons néanmoins dans la suite volontairement nous focaliser uniquement sur les impacts relatifs à la demande, en raisonnant à travers le prisme du modèle à quatre étapes. Cette modélisation est très utilisée dans le domaine de la planification de la demande de transport, et permet de rendre compte de la structure du choix de l'individu, en séparant celui-ci en quatre séquences : (i) génération, (ii) distribution, (iii) choix modal et (iv) affectation. Même si l'individu, acteur de déplacement, ne décompose effectivement pas son processus de choix de façon séquentielle, ce découpage semble bien adapté dans notre cas pour rendre compte des différents tenants de notre problématique. Ainsi, à chaque étape, les conditions météorologiques peuvent impacter les choix opérés par l'usager dans le cadre de son déplacement. Comme cela est suggéré par Koetse et Rietveld, nous nous intéresserons également à une séquence supplémentaire que constitue le choix de l'heure de départ introduite par Ortùzar et Willumsen [2] [6] [7] [8].

\section{Influence de la météorologie sur l'étape de génération}

L'étape de génération correspond au choix de l'individu de se déplacer ou de ne pas se déplacer. Elle permet donc de définir le volume de déplacement réalisé par les individus. Les impacts à analyser sont donc de deux natures : soit l'annulation du déplacement, soit la création de nouveaux déplacements [7].

L'ensemble des auteurs s'accordent à dire qu'en cas de mauvaises conditions météorologiques, certains déplacements non contraints peuvent être annulés. L'annulation $\mathrm{du}$ déplacement dépendrait en premier lieu du motif du déplacement. Pour Maze et al. comme pour Koetse et Rietveld, la viabilité de cette option serait fortement dépendante du degré de flexibilité du déplacement et des prévisions météorologiques. Ainsi, les déplacements réguliers comme les migrations pendulaires (déplacements quotidiens de type domicile-travail) seraient logiquement moins susceptibles d'être annulés que les déplacements à vocation de loisirs. Kilpeläinen et Summala ont effectivement observé et quantifié l'influence des prévisions météorologiques sur le comportement des conducteurs sur la base de deux enquêtes menées auprès de la population finlandaise entre 2001 et 2002. Une des conséquences directes de ce constat est que l'annulation de déplacements se produit surtout durant certaines périodes privilégiées. 
Les déplacements pendulaires se produisent essentiellement autour des heures de pointe du matin et du soir, en semaine. De ce fait, les annulations auront plutôt tendance a priori à se produire en dehors des heures de pointe et le week-end. Hanbali et Kuemmel ont par exemple étudié les réductions de volume du trafic routier se produisant en cas de tempêtes de neige dans plusieurs états américains. Ils aboutissent à la conclusion que la réduction de volume est dépendante de l'importance du déplacement, que celle-ci est bien moins importante durant les heures de pointe, et nettement moindre pendant les jours de semaine que pendant les week-ends, et que ces réductions sont en grande partie dues à des phénomènes d'annulation ou de report. Maki, en étudiant les volumes de trafic durant des tempêtes de neige au Minnesota, est également arrivé au même constat et suggère que les individus ont tendance à reporter leur déplacement en cas de très mauvaises conditions météorologiques, ou alors à partir plus tôt ou plus tard [9] [10] [11] [12] [13].

Si les auteurs précédemment cités se sont intéressés uniquement au domaine routier, les conclusions semblent également valables pour les autres modes de transport [14] [15]. De façon générale, la flexibilité du déplacement, le mode choisi et l'intensité du phénomène sont ainsi des facteurs à prendre en compte dans la modification des choix de déplacement dans le cas d'une météorologie défavorable. Précisons enfin que ces constats concernent aussi bien le milieu urbain qu'interurbain, même si la propension d'annulation peut être très différente. En effet, les déplacements en interurbain sont en général plus longs, avec des conditions météorologiques susceptibles de plus fortes variations qu'en urbain. De plus, les motifs de déplacement (moins de motifs domicile-travail en interurbain) et la perception du réseau sont différents (plus de sécurité, pas de mélange des flux...).

\section{Conséquences sur l'étape de distribution}

Si, à l'étape de génération l'individu décide de se déplacer, à l'étape de distribution il s'agit pour lui de choisir sa destination. En modélisation des déplacements, c'est à cette étape que l'on construit la matrice origines-destinations des déplacements [7].

Koetse et Rietveld scindent en deux catégories les impacts potentiels de la météorologie sur l'étape de distribution [10] :

- sur le court terme, ils estiment qu'en cas de conditions météorologiques défavorables, les individus pourraient changer leur destination initiale pour une destination plus proche. On peut légitimement estimer que les individus se déplaçant pour un motif « loisirs » ou « achats » sont les plus concernés par cette modification ;
- sur le long terme, Koetse et Rietveld estiment qu'une dégradation de la météorologie récurrente dans certaines régions pourrait conduire à une augmentation des coûts de déplacement (par exemple le temps de parcours) ainsi qu'à une diminution de la distance moyenne des déplacements.

Cependant, il n'existe à ce jour aucune étude sur les conséquences avérées d'un changement météorologique sur la destination d'un déplacement. Seuls Aaheim et Hauge estiment (grâce à un modèle basé sur le comportement économique) qu'il existe une réduction des distances parcourues avec l'augmentation des précipitations, et soulignent que celle-ci doit surtout concerner les déplacements de loisirs ou d'achats. La même conclusion est faite relativement à la température [4].

\section{Impacts sur la séquence du choix modal}

L'étape du choix modal correspond au choix du mode de transport à utiliser pour effectuer le déplacement entre une origine et une destination. En planification, on associe à cette séquence autant de matrices origines-destinations qu'il y a de modes possibles de transport [7].

Selon Hanson et Hanson, le changement de mode et l'inflexibilité de certains déplacements sont étroitement liés. En effet, en cas d'inflexibilité du déplacement (exemple du trajet domicile-travail), le changement de mode serait, selon eux, le seul véritable choix qui s'offre à un individu en tant qu'acteur de déplacement, le choix de l'itinéraire mis à part. C'est pour cette raison qu'un certain nombre d'études se concentrent en particulier sur les migrations pendulaires [14].

Khattak et De Palma ont réalisé une grande étude sur la modification des choix de déplacement domiciletravail des Bruxellois en cas de mauvaises conditions météorologiques. Sur l'ensemble des personnes interrogées, seules les personnes utilisant la voiture sur au moins une partie de leur trajet ont été prises en compte. Sur cette population, $27 \%$ déclarent que l'influence de la météorologie est très importante ou importante sur le choix de leur mode de transport (contre $61 \%$ pour le changement de l'heure de départ et $36 \%$ pour la modification du trajet). Ce pourcentage faible, comparé aux autres options, est néanmoins à relativiser puisque l'on ne considère que des automobilistes et que ce mode est relativement peu vulnérable aux mauvaises conditions météorologiques comparé à d'autres modes [16].

Une étude comparable menée en mars 1994 sur les migrations pendulaires dans l'agglomération genevoise par De Palma et Rochat (1999) montre des résultats assez similaires. Sur $40 \%$ des individus qui déclarent que la météo 
a une influence sur les conditions de leur déplacement, ils sont $54,7 \%$ à considérer que celle-ci à une influence très importante $(32,9 \%)$ ou importante $(21,8 \%)$ sur le choix du mode de transport. Ce pourcentage, plus élevé que dans le cas bruxellois, peut s'expliquer par la plus grande variété des utilisateurs des différents modes dans l'échantillon de base. Dans une autre étude, Nankervis relève que $25 \%$ des cyclistes de son échantillon changerait de mode de transport en cas de mauvaises conditions météo, et que parmi eux $18 \%$ se redirigeraient vers les transports en commun, les autres vers la voiture particulière. L'enquête de Nankervis prête néanmoins à caution puisque son échantillon est uniquement composé d'étudiants de la ville de Melbourne, qui n'ont, de son propre avis, pas forcément les mêmes possibilités de changement de mode que les autres catégories d'usagers [17] [18].

Bergström et Magnusson ont, quant à eux, étudié les reports qui peuvent s'opérer entre la voiture et le vélo notamment en hiver et relèvent ainsi une nette différence dans le choix du mode de transport selon la saison. Le nombre de déplacements en voiture augmenterait de $27 \%$ en hiver par rapport à l'été tandis que le nombre de déplacements à vélo diminuerait de $47 \%$. Sabir et al. ont également dressé le même constat dans le contexte néerlandais [5] [19].

Certains auteurs émettent néanmoins des réserves sur l'importance du report. Aaheim et Hauge se sont par exemple intéressés aux habitudes de déplacement des habitants de Bergen en Norvège. Les conclusions de l'étude suggèrent que l'influence de la météo (température, pluie et vent) sur le transfert modal entre voiture particulière et vélo, marche à pied ou transport en commun existerait, mais serait plus limitée. À partir d'une modélisation de nature économique, ils montrent que le vent est le paramètre présentant l'impact le plus significatif sur le choix du mode de transport, mais que son influence dépend du motif du déplacement. De plus, ils relèvent là aussi une différence selon les motifs de déplacements. La marche et le vélo augmenteraient avec la température pour des trajets domicile-travail aux dépens des transports en commun et de la voiture. De fortes précipitations feraient décroître les déplacements domicile-travail réalisés à pied ou à vélo, surtout au bénéfice de la voiture particulière alors que la fréquentation des TC resterait inchangée. Les déplacements de loisirs sont affectés de la même façon, mais les réactions en cas de pluie sont beaucoup plus fortes dans ce cas. Par contre, le choix du mode pour les déplacements avec motif achats dépend majoritairement des précipitations. Une augmentation des températures réduit l'usage du vélo et la pratique de la marche et augmente l'utilisation des transports en commun et de la voiture. Cependant, ces résultats doivent s'interpréter avec prudence, car Aaheim et Hauge utilisent des données météorologiques journalières au lieu de données horaires [4].

\section{Effets sur l'étape d'affectation}

On trouve peu d'études sur les effets de la météorologie sur le choix d'itinéraires dans la littérature. Cette carence est sans doute liée au fait que les décisions de changement de mode ou de modification de l'heure de départ sont les deux principaux leviers utilisés par les usagers. Khattak relevait dans le cas d'un blizzard à Chicago une augmentation de la propension de modifications des choix habituels de déplacement, dont le trajet. Il estime alors à $80 \%$ le nombre d'automobilistes qui changent une de leurs décisions, sans précision sur le pourcentage de ceux qui changent de trajet. Néanmoins, ce pourcentage est à relativiser compte tenu de la nature exceptionnelle et de l'importante intensité du phénomène. Il précise enfin que les individus mâles, « agressifs et anxieux », qui choisissent habituellement leur route en cours de déplacement et qui connaissent des routes alternatives où le temps de parcours durant le phénomène est plus court que celui sur sa route habituelle étaient plus susceptibles de changer de route que les autres [20].

Dans leur étude concernant l'impact de la météo sur les habitudes de déplacement des Bruxellois, Khattak et De Palma se sont interrogés sur les changements d'itinéraire. Comme pour le changement de mode, les personnes sondées devaient évaluer les effets de la météo sur leur propension à changer de route en donnant une note sur une échelle de 1 à 5 (d'inexistant à très important). Parmi ceux qui estiment que les conditions atmosphériques ont un impact sur la façon qu'ils ont de se déplacer (environ $54 \%$ des automobilistes effectuant un trajet domicile-travail), $35 \%(\mathrm{~N}=131$ sur $\mathrm{N}=375$ ) estiment qu'il y a un effet sur le changement d'itinéraire (14\% considèrent l'effet très important et $21 \%$ le considèrent important) [16].

On le voit dans la partie suivante, ce pourcentage est néanmoins faible, devant celui lié au changement de l'heure de départ. Les auteurs avancent plusieurs raisons à cela. Tout d'abord, ils estiment que le changement de route n'est pas forcément une solution car dans le cas de conditions météorologiques dégradées les différents itinéraires sont affectés de la même façon. Ce point est d'autant plus vrai que les trajets domicile-travail se font généralement sur des distances assez courtes, c'est-à-dire dans une aire géographique limitée où les conditions météorologiques ont peu de chance de varier. Ensuite, ils estiment que la décision de changer de route n'est pas forcément la plus facile à prendre, notamment parce qu'il faut avoir le choix de routes alternatives et disposer d'informations sur ces itinéraires (sur les conditions de circulation, temps de parcours habituel...). Ce point est néanmoins discutable puisque $57 \%$ des individus de l'échantillon initial disent avoir le choix relatif aux itinéraires pour se rendre à leur travail. Enfin, les conséquences d'un changement de route sont relativement imprévisibles, surtout comparées à la décision du changement de l'heure de départ. 
Enfin, et de la même manière que pour le changement de mode, Khattak et De Palma ont tenté de réaliser un modèle Probit ordinal permettant d'expliquer la propension des automobilistes à changer de route en cas de météo défavorable en fonction de diverses variables (salaire, temps de parcours, flexibilité dans les horaires de travail...). Cependant, pour les raisons évoquées précédemment, ce modèle n'est pas statistiquement significatif.

L'étude similaire menée en mars 1994 sur les déplacements domicile-travail à Genève par De Palma et Rochat (1999) montre des résultats assez similaires. Sur les $40 \%$ $(\mathrm{N}=320)$ des individus qui déclarent que la météo a une influence sur les conditions de leur déplacement, ils sont $49,6 \%(\mathrm{~N}=159)$ à penser que celle-ci a une influence sur le choix du trajet (26,8\% estiment l'influence très importante, $22,8 \%$ importante). Même si ce chiffre est un peu plus important que celui de Bruxelles, il est, au sein de l'étude genevoise, légèrement plus faible que celui obtenu pour l'influence sur le changement de mode et que celui sur le changement d'heure de départ - qui est à nouveau largement devant. Il faut néanmoins être prudent dans la comparaison entre les deux études, car on ne considère pas ici uniquement des automobilistes (comme dans l'étude bruxelloise).

Signalons enfin que Maze et al. ont mis en évidence la propension de plusieurs entreprises de transport de fret à changer d'itinéraire en cas de tempêtes de neige dans l'Iowa (États-Unis). Ces dernières n'ont pas la possibilité d'annuler, voire de reporter, un déplacement en raison des contraintes liées à cette activité. Néanmoins, ces changements d'itinéraire sont a priori liés aux fermetures de certaines routes principales, puisque les routes alternatives empruntées sont jugées moins bonnes en termes de sécurité et ont des normes de conception moins évoluées. La plupart des entreprises interrogées relèvent d'ailleurs que cette solution n'est absolument pas pratique, et qu'il est en général (même en cas de non-fermeture de la route principale) pratiquement impossible de changer d'itinéraire, car les routes alternatives sont affectées par les mêmes conditions climatiques et sont pour la plupart plus lentes. Quelques rares entreprises préfèrent même attendre la réouverture de la route principale plutôt que de changer de route. Précisons enfin que pour ces entreprises, changer d'itinéraire est pratiquement la seule solution, l'annulation ou le report du déplacement engendrant souvent des pertes financières importantes, et le changement de mode se révélant pratiquement impossible [21].

\section{Modification de l'horaire de départ}

Cette partie aborde une séquence qui pourrait s'ajouter lors de la modélisation des déplacements à celles du modèle à 4 étapes : le choix de l'heure de départ. Il s'agit de voir si, et dans quelle mesure, la modification de l'heure de départ (anticipation ou report du déplacement) constitue une réaction possible de l'usager en cas de conditions météorologiques défavorables [8].

Selon Koetse et Rietveld, le changement de l'heure de départ dépendrait surtout de la nature du déplacement, et donc de sa flexibilité. On retrouve la même réflexion que celle faite à propos de l'annulation, même si dans ce cas la flexibilité doit évidemment être supérieure. Selon eux, les déplacements non prévus (dans le sens où ils ne font pas partie d'une routine journalière, par exemple emmener ses enfants à l'école) seraient facilement reportables, alors que les déplacements fixes, comme les migrations pendulaires, ne le seraient pas. Richardson fait d'ailleurs, dans son étude, la distinction entre les déplacements réalisés quelles que soient les conditions météorologiques (aller au travail, aller à l'école, se rendre à un rendez-vous professionnel. . .) et ceux pouvant être facilement reportés (loisirs, achats non vitaux...). Il arrive ainsi à la conclusion qu'en cas de pluie une partie des trajets à vélo non essentiels seraient reportés, alors que les autres seraient tout de même réalisés. Kilpeläinen et Summala se sont, eux, basés sur les résultats d'une régression logistique binaire sur l'influence des prévisions météorologiques sur le comportement des conducteurs finlandais. D'après ce modèle, quand les prévisions météorologiques sont considérées très mauvaises par le conducteur le déplacement a plutôt tendance à être un déplacement pendulaire ou un déplacement lié au travail [10] [11] [15].

Khattak et De Palma se sont également intéressés dans leur étude bruxelloise à l'influence de la météorologie sur l'heure de départ des déplacements domicile-travail. Sur l'ensemble des automobilistes estimant que les conditions climatiques ont un impact sur la façon qu'ils ont de se déplacer (environ $54 \%$ des automobilistes effectuant un trajet domicile-travail), $29 \%$ déclarent que l'influence de la météo sur l'heure de départ est très importante et $32 \%$ la considèrent comme importante. Cette solution est ainsi bien plus privilégiée que le changement de mode $(27 \%)$ ou la modification d'itinéraire (36\%). Les auteurs interprétent cet important écart par à la fois une meilleure flexibilité de l'heure de départ, mais aussi par le caractère moins perturbateur du décalage d'horaire et sa plus facile mise en place. Les automobilistes allant travailler seraient ainsi prêts à aménager leur emploi du temps de façon à arriver à l'heure habituelle à leur travail : en partant plus tôt, ils compensent la perte de temps sur la route subie à cause des mauvaises conditions météo. Un autre avantage cité par ces auteurs est que les conséquences d'un changement de l'heure de départ sont globalement prévisibles, c'est-à-dire que l'individu arrivera plus tôt s'il part plus tôt [16].

L'étude menée auprès des habitants de Genève (De Palma et Rochat, 1999) conduit à des conclusions pratiquement 
similaires : le changement de l'horaire serait le facteur le plus sensible à des conditions météorologiques défavorables avec 42,9 \% qui trouvent cet aspect très important et $29,8 \%$ important (soit un taux de 72,7 \% légèrement supérieur à celui trouvé à Bruxelles, contre $54,7 \%$ pour le choix du mode et $49,6 \%$ pour le choix de la route).

\section{Importance des facteurs culturels et régionaux}

Comme le soulignent Khattak et De Palma, même s'il est intéressant de comparer les résultats de différentes enquêtes à ce sujet afin de constituer une base de connaissances solide, la synthèse des résultats reste assez complexe [16]. Il existe en effet d'importantes différences entre les études, d'une part méthodologiques (manière de poser les questions, composition des échantillons...), mais aussi et surtout contextuelles.

L'influence de la météo sur les comportements est différente selon les caractéristiques des individus au sein d'une région, mais aussi et surtout entre les régions à travers le monde - les différences culturelles. À travers le monde, ce ne sont pas forcément les mêmes catégories de personnes qui effectuent les mêmes types de déplacements et l'importance accordée à un trajet varie (femmes actives ou non, flexibilité et amplitude des horaires différentes, etc.).

Rietveld et Daniel (2004) prennent pour illustrer cela l'exemple du statut du mode cyclable à travers le monde. Selon les pays, celui-ci serait ainsi considéré comme un mode de transport destiné aux classes pauvres, ou bien à l'extrême comme un mode sportif qui reflète un style de vie très actif. Ainsi les parts modales diffèrent généralement beaucoup selon les pays. Dans les pays européens la part des modes de transport non motorisés par rapport au nombre total de kilomètres parcourus varie de $3 \%$ à $10 \%$. Les différences seraient même plus importantes si l'on considère la part des déplacements par personne par jour, s'étalant de $10 \%$ à $48 \%$. Les Pays-Bas sont souvent cités en exemple, avec $27 \%$ de part modale pour le vélo contre $4 \%$ pour la France, selon la Fédération française des usagers de la bicyclette en 2009 [22].

Les différences climatiques entre les régions changent également la perception du caractère dégradé des conditions météorologiques. Comment comparer par exemple les Finlandais, qui connaissent des hivers très froids $\left(-30{ }^{\circ} \mathrm{C}\right.$ en Laponie n'a rien d'exceptionnel) et qui durent très longtemps (entre 5 mois dans le Sud et 7 mois dans le Nord) aux Français ? Les uns, habitués à des chutes de neige fréquentes ont sans doute un seuil plus fort de sensibilité à la neige que les autres - par exemple une légère chute de neige pour un Français pourrait passer comme anodine et sans conséquence pour un Finlandais.
De même, la réaction des exploitants sera différente en raison de l'expérience, et cela influe également sur le comportement de l'usager, et donc sur la demande. Dans l'étude de Winters et al., les variables individuelles et climatiques du modèle final expliquent ainsi respectivement $24 \%$ des différences entre villes canadiennes dans la pratique quotidienne du vélo (motif domicile-travail) [23].

Il faut donc se garder d'effectuer des comparaisons formelles de ces études, et effectuer seulement des comparaisons qualitatives en conservant bien en tête le contexte régional et culturel dans lequel s'inscrivent ces études.

\section{Quantification des effets sur la demande de trafic}

\section{Difficultés liées à la mesure de la demande}

La demande de trafic routier est en général assez difficile à appréhender. Afin de déterminer l'influence de la météorologie sur celle-ci, on se base sur la mesure de volumes de trafic obtenus par des comptages véhiculaires. On peut par exemple typiquement comparer le volume moyen d'une journée où le temps est $\mathrm{sec}$ avec le volume moyen sur cette même route par temps pluvieux pour examiner les conséquences de la pluie. Les variations observées ne correspondent alors pas forcément à une modification du volume de la demande à proprement parler (augmentation ou diminution). Plusieurs facteurs interviennent en effet dans la composition de cette variation. Ces facteurs sont listés en Figure 2.

La mesure des débits, notamment routiers, rend donc uniquement possible de déterminer si la météo a bien un effet sur le trafic, et ensuite d'inférer des hypothèses sur les changements de comportement qui expliquent ces variations. Il faut toujours comparer la période pendant laquelle on considère le pourcentage de variation de trafic avec la période où l'événement météo a lieu. Il convient donc dans la suite d'être vigilant vis-à-vis des différents auteurs cités, afin de garder un nécessaire regard critique sur les données et les conclusions qu'en tirent les uns et les autres.

\section{Impact de la pluie sur les volumes routiers}

Si plusieurs auteurs se sont penchés sur le problème de l'influence de la météorologie sur la demande et ont, comme nous allons le voir, mis en évidence des variations de volume de trafic liées à des phénomènes météorologiques, ils ne sont pas d'accord sur l'intensité de ces phénomènes (amplitude de la variation de trafic). 


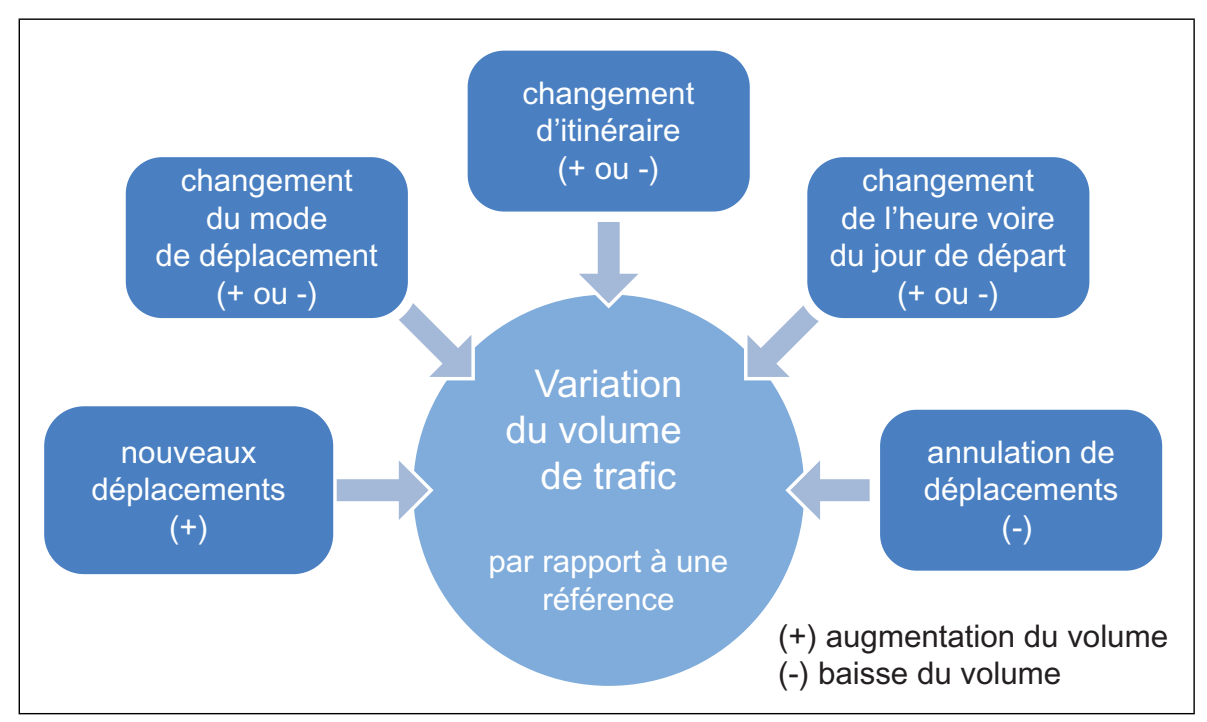

Fig. 2 Différentes causes comportementales d'une variation de volume de trafic

En 1965, le Road Research Laboratory est l'un des premiers à traiter des effets de la pluie sur les volumes de trafic. Il note ainsi durant plusieurs mois pluvieux des réductions de débits. Codling est cependant le premier à avoir estimé ces réductions en étudiant l'impact de la pluie sur les accidents en Grande-Bretagne à partir de données récoltées sur 50 sites entre les années 1969 et 1970. En raison de l'absence de données météo, Codling s'est basé sur les procès-verbaux des accidents pour classer les jours pluvieux et les jours secs. Il trouve ainsi qu'en semaine la réduction moyenne du volume de trafic les jours pluvieux se situe à peine au-dessus des $1 \%$. Par contre, le dimanche cette réduction est bien plus élevée avec en moyenne une baisse de 7,6\% en septembre et de 17,8\% pour le mois de janvier. Codling a répété également le même exercice avec des données sur le trafic londonien de 1969 et releva une réduction de $2 \%$ les jours pluvieux en semaine Il relève enfin à partir de ces deux études que la répartition de ces baisses n'est pas uniforme selon les jours de la semaine et les mois de l'année. Ces résultats prêtent néanmoins à caution : les échantillons sont à chaque fois très faibles (16 jours pluvieux dans le premier cas et 7 pour le second) et l'intensité du phénomène n'est pas du tout abordée. Néanmoins, ces premiers résultats ont permis d'ouvrir la voie à des recherches plus poussées [24].

C'est ainsi que, plus de vingt ans plus tard, Changnon s'est interrogé sur les effets des pluies survenant l'été (saison au cours de laquelle les individus s'attendent moins au phénomène a priori) sur l'accidentologie et sur les volumes de trafic dans la zone urbaine de Chicago (dans l'Illinois, Amérique du Nord). À partir de données récoltées sur une période de trois ans, il arrive à la conclusion que la pluie a un impact négligeable en semaine sur le trafic des autoroutes de l'agglomération (inférieur à $1 \%$ ), mais fort les week-ends pluvieux (décroissance du trafic d'environ 9\%). Néanmoins, le manque de précisions sur la méthodologie de Changnon empêche de tirer plus de conclusions (nombre de stations de comptage, période d'agrégation des données, qualité des données météo, définition des jours pluvieux...) [25].

La même année, Hogema, étudie les déplacements sur les autoroutes A16 et A59 au niveau de la ville de Breda (Pays-Bas) de novembre 1991 à mars 1994, et conclut qu'il n'y aurait pas de différence significative entre le volume journalier moyen les jours de pluie et celui des jours secs - pas de report modal majeur vers la voiture en cas de pluie. Hogema utilise de plus, pour cette étude, une comparaison des échantillons des volumes des jours pluvieux et secs grâce à un test de Student, avec plusieurs critères de définitions des jours pluvieux : pluie faible (entre 0,1 et $1 \mathrm{~mm} / \mathrm{h}$ ), modérée (entre 1 et $5 \mathrm{~mm} / \mathrm{h}$ ) et forte $(>5$ $\mathrm{mm} / \mathrm{h}$ ). On peut cependant regretter la non séparation des jours de semaine des jours du week-end. De plus, l'auteur reconnaît lui-même plusieurs limites de son étude, comme l'utilisation d'un échantillon faible de jours pluvieux $(\mathrm{N}=$ 64), une mesure du niveau de pluie éloignée d'environ 12 $\mathrm{km}$ des mesures du trafic, un intervalle de mesure des pluies important (1 h) [26].

Hassan et Barker étudient de leur côté l'impact d'une météorologie dégradée ou de conditions météo extrêmes sur les volumes de trafic de la région du Lothian, en Écosse sur la période 1987-1991 (une station sur l'autoroute M8 représentative de l'ensemble des routes de la région). Ils établissent à partir des données un modèle simple de prévision de trafic (basé sur des variations annuelles, mensuelles et journalières) et un modèle de prévisions 
à plusieurs variables météorologiques, dont les chutes de neige. Ils identifient ensuite les $10 \%$ des jours avec les plus fortes valeurs et les plus faibles valeurs de ces variables (les extrêmes), mais aussi les $10 \%$ les plus atypiques (valeurs inhabituelles pour la saison). Il leur est alors possible d'étudier l'effet de pluies plus importantes ou moins importantes que celles attendues pour la saison, mais aussi de pluies jugées « extrêmes », c'est-à-dire les plus fortes pluies de l'année [27].

Les résultats de l'étude leur permettent de dire que des liens statistiquement significatifs existent entre la pluie et le trafic pour la région Lothian. Comme Changnon, ils estiment que la pluie aurait un effet faible en semaine $(-0,82 \%$ de réduction moyenne du trafic journalier pour des pluies plus fortes que de saison, idem pour les jours où les pluies sont les plus fortes de l'année, au risque de $10 \%$ ), mais important le week-end $(-4,73 \%$ pour des pluies plus fortes que de saison et $-4,6 \%$ pour les plus fortes pluies, au risque de $1 \%)$. Al Hassan et Barker suggèrent ainsi qu'il n'y aurait pas de changement modal majeur en semaine et qu'il n'est pas nécessaire de s'occuper du peu de jours où il y a de la pluie sur le réseau. Il pourrait cependant y avoir, là aussi, sous-estimation du phénomène à cause de l'utilisation de moyennes journalières. En effet, il ne pleut en général pas toute la journée, mais juste sur des courtes périodes, l'effet direct de la pluie sur le trafic pourrait ainsi se retrouver « noyé » par les volumes de trafic normaux du reste de la journée [25].

Pour Chung et al., qui étudient les effets de la pluie sur le réseau autoroutier de Tokyo, les effets existent, mais sont également faibles en semaine $(-2,9 \%$ de trafic en moyenne pour les jours pluvieux où la quantité de pluie tombée est supérieure à $13 \mathrm{~mm}$ ), mais fort le week-end $(-7,9 \%$ en moyenne pour les samedis pluvieux et $-5,2 \%$ pour les dimanches pluvieux, le critère de $13 \mathrm{~mm}$ étant conservé). Une "sensibilité plus forte à la pluie » est avancée pour les déplacements du week-end, en raison de la forte proportion de déplacements «loisirs » le samedi en tête, suivi du dimanche. Plusieurs seuils permettant de définir un jour pluvieux ont été testés et permettent de constater l'importance de l'intensité du phénomène : à titre d'exemple pour un samedi, on passe d'une réduction avoisinant les $4 \%$ si on considère tous les jours avec des pluies de niveaux supérieurs à $0 \mathrm{~mm} /$ jour, mais on atteint pratiquement $15 \%$ dans le cas de jours où les quantités sont supérieures ou égales à $30 \mathrm{~mm} /$ jour [28].

Bien que l'étude de Chung et al. porte sur un volume de données important (trafics journaliers entre 1998 et 2004 sur plusieurs stations de comptage, avec 216 jours considérés comme pluvieux sur les six ans), elle prend seulement en compte une station météo pour l'ensemble du réseau. Or celui-ci est étendu $(283 \mathrm{~km})$ et l'on comprend bien qu'il pourrait par exemple y avoir de la pluie au nord du réseau sans qu'il y en ait au sud. C'est pour pallier ce phénomène qu'un seuil important (13 mm/jour) a, selon les auteurs, été introduit afin d'avoir plus de chances d'observer de la pluie à la fois sur la station centrale et sur les autres stations du réseau. Avec ce seuil, le biais est limité, mais demeure néanmoins.

Enfin, Keay et Simmonds ont étudié les effets de plusieurs facteurs météorologiques sur les trafics de la ville de Melbourne, en Australie. Cette étude est sans doute à ce jour une des plus complètes, car elle sépare pour la première fois les effets selon les périodes de la journée (le jour de $6 \mathrm{~h}$ à $18 \mathrm{~h}$ et la nuit de $18 \mathrm{~h}$ à $6 \mathrm{~h}$ ), mais aussi selon les saisons. L'étude porte sur deux voies rapides de l'aire métropolitaine de Melbourne, la Southeastern Freeway et la Westgate Freeway, grâce à des mesures intermittentes des débits faites entre 1989 et 1996. À partir de modèles de régression, Keay et Simmonds ont estimé que la pluie était l'événement météorologique ayant la plus forte corrélation avec les variations de débit (-1,29\% pour un jour humide, c'est-à-dire un jour où plus de $0 \mathrm{~mm}$ de pluie est tombé, par rapport à un jour sec) [29].

Ils ont également établi l'existence d'une relation négative entre le volume de trafic et la quantité de pluie tombée $(-0,08 \%$ par $\mathrm{mm}$ de pluie tombée en $24 \mathrm{~h}$ pour un jour humide par rapport à un jour sec). La prise en compte, en tant que variables, de classes séparant les volumes de pluie tombés en $24 \mathrm{~h}$ permet d'estimer qu'il y aurait une tendance à de plus fortes réductions de trafic en cas de pluies abondantes. En général, la réduction la plus importante est pour la classe $2-10 \mathrm{~mm} /$ jour où celle-ci vaut entre $2 \%$ et $3 \%$. L'impact est le plus fort en hiver et au printemps : sur une journée entière, la réduction de trafic serait de 1,35\% les jours pluvieux en hiver et de $2,11 \%$ les jours pluvieux au printemps. Ces réductions restent faibles et dans la continuité de celles trouvées dans la bibliographie. Ces valeurs sont assez proches de celles trouvées par Codling, Changnon, Hassan et Barker et Chung et al. [24] [25] [27] [28].

Keay et Simmonds concluent également sur une influence plus forte de la pluie la nuit qu'en journée. En effet, ils aboutissent à une réduction statistiquement significative de $1 \%$ en journée les jours pluvieux et de 2,61\% les nuits pluvieuses. En journée, les impacts sont égaux à $-1,86 \%$ en hiver et $-2,16 \%$ au printemps (les résultats pour les deux autres saisons ne sont pas significatifs). La nuit, les impacts sont significatifs quelle que soit la saison, mais ils sont les plus forts au printemps $(-2,91 \%)$ et en été $(-2,17 \%)$. Les auteurs notent également que la pluie pendant la période $12 \mathrm{~h}-18 \mathrm{~h}$ affecte de façon statistiquement significative le trafic en hiver (entre $-0,45 \%$ et $-0,70 \%$ ), de même que pour la période $21 \mathrm{~h}-24 \mathrm{~h}$ en hiver $(-0,65 \%)$ et en été $(-1,51 \%)$. 
Ces derniers traduisent ce phénomène par une flexibilité plus importante du choix de déplacement la nuit, et une propension des gens à donc ne pas se déplacer en raison de la pluie et du risque routier qui en résulte.

\section{Effets de la neige sur la demande de trafic}

Une partie de la littérature s'intéresse aux variations de volume du trafic routier lors de phénomènes extrêmes comme des tempêtes de neige (effets supposés plus facilement visibles, en général sur les routes de type autoroute ou voies rapides urbaines) qui sont plus fréquents dans les pays d'Europe du nord ou d'Amérique du nord. Précisons que toutes les études sur l'impact de la neige se focalisent sur le domaine routier, donc principalement sur les modes automobiles. Les autres modes, comme la marche et le vélo - les 2RM ne sont a priori pas abordés. Pourtant on peut supposer, compte tenu de l'impact de la pluie vu précédemment, que ces modes sont très impactés par la neige, et qu'un volume important de report modal est susceptible de se produire - annulation, report ou anticipation des déplacements.

Hanbali et Kuemmel sont les premiers à s'intéresser aux réductions de volumes de trafic autoroutiers engendrées par les tempêtes de neige (survenues en 1989, 1990 et 1991) pour quatre états nord-américains (Illinois, Minnesota, New York et Wisconsin). Pour chaque tempête, le volume de trafic horaire est comparé au volume de trafic horaire dit « normal » (même jour de la semaine, à la même heure, le même mois, la même année). Hanbali et Kuemmel tirent plusieurs conclusions de leur étude. Premièrement, ils relèvent que plus la tempête est violente et plus la réduction de débit générée est forte. Deuxièmement, les auteurs notent que la réduction serait plus importante le week-end que la semaine, en distinguant selon l'intensité des chutes de neige (par exemple pour des chutes inférieures à $25 \mathrm{~mm}$ on trouve $7 \%$ à $17 \%$ en semaine, et de 19 à $31 \%$ le week-end, pour des chutes entre 150 et $225 \mathrm{~mm}$ la réduction est de $35 \%$ à $49 \%$ en semaine et de $41 \%$ à $51 \%$ le week-end) [12].

Les auteurs mettent ensuite en évidence un lien entre cette diminution et l'importance du déplacement (plus celle-ci est importante, comme pour les migrations pendulaires, et moins la réduction moyenne est élevée). Ce lien serait sûrement une des raisons des différences de réduction observées entre les heures de pointe et les heures creuses.

La même année, Hassan et Barker considèrent également la neige dans leur étude sur l'impact d'une météo dégradée sur les volumes de trafic de la région du Lothian. En semaine, ils obtiennent ainsi une réduction moyenne du trafic d'environ $10 \%$. Cette réduction est plus importante le week-end avec environ $-15,3 \%$ en moyenne par rapport à la situation normale. Comme pour la pluie, aucune distinction n'est par contre faite dans l'étude sur l'intensité des phénomènes neigeux puisque les auteurs ne raisonnent que sur des moyennes journalières. Il pourrait donc y avoir, là aussi, sous-estimation du phénomène puisqu'il est fort probable qu'il ne neige pas toute la journée et que des journées soient prises en compte alors que très peu de neige tombe [27].

Maki s'intéresse également cette année-là aux réductions liées aux tempêtes de neige dans le Minnesota (Amérique du Nord), afin d'étudier l'opportunité de mise en place d'un plan de feu particulier en cas de conditions météo dégradées. L'étude concerne uniquement une portion de route de $5 \mathrm{~km}$ environ de l'autoroute 36 (plutôt à considérer comme une route nationale avec une limitée à $105 \mathrm{~km} / \mathrm{h}$ et 5 feux de signalisation) et les routes qui la croisent. Les volumes relevés durant les tempêtes sont entre $15 \%$ et $20 \%$ plus bas que ceux relevés habituellement pendant la période $15 \mathrm{~h}-20 \mathrm{~h}$, et $15 \%$ à $30 \%$ plus bas sur le créneau de pointe $17 \mathrm{~h}-18 \mathrm{~h}$. Cette diminution est attribuée par Maki à des personnes retardant ou avançant leur déplacement travail-domicile afin d'éviter les risques liés à la tempête ou à des individus qui annulent des voyages jugés non-essentiels à cause de la météo. Ces résultats doivent néanmoins être considérés surtout à titre indicatif : seulement trois jours de conditions météo dégradées furent pris en compte et comparés à deux jours dits «normaux », et ceci sans précision sur le volume de neige tombée même si a priori il doit être assez important pour être classé dans les tempêtes de neige [13].

Fridstrøm s'est, pour sa part, penché sur l'influence des facteurs saisonniers sur le nombre de kilomètres parcourus (mesuré en veh.km). À partir de données collectées chaque mois entre janvier 1973 et décembre 1994 sur des routes (en général des autoroutes) de toutes les provinces norvégiennes, Fridstrøm a réalisé une régression non linéaire (modèle de Box-Cox) qui explique la moyenne totale du nombre de kilomètres parcourus en fonction de variables météo comme le nombre de jours de neige dans le mois. L'auteur trouve une élasticité de $-0,025$ entre ces deux éléments, et estime ainsi que les chutes de neige ont tendance à faire chuter le volume de trafic routier, mais pas de façon drastique. Il estime à partir du modèle à $5 \%$ la réduction totale de trafic pour un mois comprenant 10 jours de neige, par rapport à un mois sans neige. Il précise néanmoins la difficulté d'extrapolation de ces résultats à d'autres pays, car la neige est très commune en Norvège : à titre d'exemple, dans son échantillon la neige intervient lors de plus de la moitié des mois retenus. Par contre, il conclut que l'influence du pourcentage des jours de neige où les chutes sont de forte intensité (au-dessus de $5 \mathrm{~mm}$ sous forme liquide) sur le nombre de kilomètres parcourus n'est pas significative d'après le modèle [30].

De leur côté, Knapp et Smithson ont étudié les effets de 64 tempêtes de neige sur plusieurs autoroutes de l'Iowa 
(Amérique du Nord, notamment l'Interstate 35, l'Interstate 80 et l'Interstate 380) durant la période 1995-1998. En plus de plusieurs critères qui selon eux caractérisent ces tempêtes (précipitations neigeuses, température de l'air en dessous de $0{ }^{\circ} \mathrm{C}$, chaussée humide et température de la chaussée inférieure à $0{ }^{\circ} \mathrm{C}$ ), ils ne considèrent que les phénomènes d'une durée supérieure à $4 \mathrm{~h}$ et avec une intensité de chute de neige supérieure à $0,51 \mathrm{~cm} / \mathrm{h}$. Les réductions moyennes des volumes de trafic (comparaison par rapport à la moyenne des volumes des mêmes jours de la semaine du même mois, données horaires) liées à la tempête s'étalent de $16 \%$ à $47 \%$ avec une moyenne totale de $29 \%$ (avec un intervalle de confiance allant de $22,3 \%$ à 35,8\%). Les auteurs soulignent avant tout, en plus des baisses de débits mises en évidence, l'amplitude très importante des variations observées. Ils ont de plus réalisé une régression linéaire et mis en évidence une relation entre le pourcentage de réduction de trafic et le volume de neige tombé en centimètres ainsi que le carré de la vitesse maximum du vent [31].

Maze et al. ont également étudié le cas de l'Interstate 35 avec l'impact des jours de neige sur le trafic (pour Maze et al., un jour de neige est un jour pendant lequel plus de $2,5 \mathrm{~cm}$ de neige sont tombés). Les jours de neige avec une bonne visibilité et un vent faible, ils ont pu observer une réduction moyenne du trafic d'environ $20 \%$. Dans le cas de jours de neige avec une faible visibilité (inférieure à $400 \mathrm{~m}$ ) et un vent fort (vitesse supérieure à $60 \mathrm{~km} / \mathrm{h}$ ), les réductions étaient plutôt de l'ordre de $80 \%$. Cette réduction est la réduction la plus forte observée par un des auteurs en la matière [9].

Enfin, Datla et Sharma considèrent pour la première fois l'impact de l'interaction du froid et de la neige sur les variations horaires de volume de trafic relevées sur 350 boucles du réseau autoroutier de l'agglomération d'Alberta, au Canada, durant la période 1995-2005. Les résultats de l'étude (régression multiple permettant d'expliquer la variation de trafic) indiquent que l'impact du froid et de la neige sur le volume de trafic varie en fonction du jour de la semaine, de l'heure du jour et de l'intensité des conditions atmosphériques. Le trafic moyen journalier décroit avec l'augmentation de la sévérité du froid et de la chute de neige. Une réduction de trafic comprise entre $1 \%$ et $2 \%$ pour chaque centimètre de neige tombé est observée quand les températures moyennes sont en dessous de $0{ }^{\circ} \mathrm{C}$. Pour les jours où il n'y a pas de précipitations, les réductions de trafic dues à un froid modéré sont de $1 \%$ et de $31 \%$ pour un froid extrême. Une réduction supplémentaire de $0 \%$ à $3 \%$ par centimètre de neige tombé survient dans le cas d'un froid extrême. Les auteurs soulignent également que les déplacements faits le week-end sont plus touchés par la baisse de trafic que ceux effectués en semaine. Comme Hanbali et Kuemmel, ils notent également l'importance du motif, puisque l'interaction neige/froid a un impact assez faible sur les trajets domicile-travail comparé aux autres motifs [3] [12].

\section{Autres impacts météorologiques et autres modes de déplacement}

Nous nous sommes focalisés uniquement sur la demande routière ; les autres modes, à l'exception des deux-roues, sont rarement étudiés. On peut citer à cette occasion Changnon, Emmerson et al., Nankervis, Goetzke et Rave ou Winters et al. [18] [23] [25] [32] [33].

De plus, la neige et la pluie sont reconnues par de nombreux auteurs comme les facteurs ayant l'impact le plus important sur les volumes de trafic, et donc potentiellement sur la demande. Cependant, certains auteurs se sont également intéressés à l'influence de plusieurs autres facteurs météorologiques sur les volumes de trafic. Certains, comme la température, la durée d'ensoleillement ou même l'éblouissement, sont plutôt étudiés sur le long terme, dans l'optique d'un effet saisonnier, alors que d'autres comme le vent, auront des impacts plutôt immédiats. Ces thèmes sont en général de préférence abordés sous l'angle de la pratique cyclable, car ce mode est $a$ priori plus sensible à ces facteurs que d'autres modes plus «protégés » comme la voiture particulière. On peut citer à ce propos Knapp et al. ou Sabir et al. pour l'effet du vent [5] [31], Hanson et Hanson pour la température [14], ou bien Auffray [34] pour l'éblouissement et Fridstrøm [30] pour l'ensoleillement.

\section{Étude de données opérationnelles en milieu interurbain et milieu urbain}

Dans cette partie, nous analysons l'impact des précipitations sur les volumes de trafic afin d'appréhender les variations de la demande en milieu périurbain à partir des données opérationnelles de la société Area et, en milieu urbain, à partir de celles recueillies sur la route $\mathrm{n}^{\circ} 3$ à Tokyo. Notre démarche peut se décomposer en plusieurs étapes, et se fonde notamment sur une analyse statistique des demandes des jours pluvieux (et des jours neigeux pour les données Area) par rapport aux jours secs.

\section{Méthodologie}

Avant toute chose, nous dressons le plan d'expérience de l'étude. Pour analyser l'impact d'une météorologie défavorable sur la demande, il est nécessaire de faire varier uniquement le facteur météorologique considéré (neige, pluie...) et de garder constants les autres facteurs 
susceptibles d'influencer les volumes de trafic. Il est également important de lister tous les facteurs autres que les facteurs météorologiques étudiés susceptibles d'influencer les volumes de trafic, par exemple :

- la période du jour (jour/nuit) ;

- la période de la semaine (le volume de trafic le lundi n'est pas nécessairement le même que celui le mardi, et encore moins que celui du week-end, notamment à cause de la différence dans les motifs de déplacement) ;

- la période de l'année (vacances scolaires, jours fériés...);

- les conditions globales de circulation (situation fluide ou saturée, événement exceptionnel de type accident. ..);

- etc.

Il convient ainsi de faire des comparaisons sur des plages de données comparables entre elles, et d'identifier toutes les causes potentielles de variation de la demande de déplacement et donc des volumes. À titre d'exemple, pour étudier l'influence de la pluie, il n'est pas concevable de comparer un dimanche pluvieux à un mardi sec, puisque la demande d'un dimanche sec n'est a priori déjà pas comparable à celle d'un mardi sec. Précisons enfin, qu'idéalement il nous faudrait des données sur l'ensemble des modes de déplacement et sur un ensemble de routes afin d'établir ensuite les causes des variations de demande observées (report vers un autre itinéraire, changement de mode...). Néanmoins, nous ne disposons que de données sur un axe routier. Nous nous concentrons donc uniquement sur la demande de trafic sur un axe routier spécifique, en distinguant toutefois la part de l'activité poids lourd parmi le trafic global dans le cas des données de l'A43.

\section{Présentation des axes étudiés et des données}

\section{La section Lyon-Chambéry de l'A43}

Comme nous l'avons dit en introduction, les données de trafic utilisées dans cette étude proviennent, dans le cas interurbain, des stations de comptage véhiculaire situées sur l'A43 de la société Area. Sur cette autoroute d'un peu plus de $200 \mathrm{~km}$ au total et qui relie Lyon à Modane, nous intéressons uniquement à la section Lyon-Chambéry, d'une longueur de $90 \mathrm{~km}$ environ. Cette section est assez atypique puisqu'en semaine elle est utilisée par un certain nombre d'habitants de Chambéry et de Grenoble pour aller travailler à Lyon (et vice et versa), mais est également le week-end l'itinéraire privilégié vers les pistes de ski de Savoie et de Haute-Savoie ( $c f$. Figure 3).

Les données de deux stations de comptage implantées sur cette section sont analysées : celles de la station localisée au point kilométrique 21 (PK 21) à la barrière de péage de
Saint-Quentin-Fallavier à proximité de Lyon, et celle du PK 82 sur la section Aiguebelette-Chambéry. Les données transmises sont des comptages (débits) à la fois pour l'ensemble des véhicules s'écoulant sur l'autoroute (les véhicules légers ou VL uniquement ou les poids lourds uniquement, dans les deux sens de circulation et pour la période de janvier 2008 au début du mois de mai 2010). Pour les besoins de l'étude, nous ne conservons que les données de la première station dans le sens Lyon-Chambéry et les données de la seconde dans le sens Chambéry-Lyon afin de bien appréhender la demande de trafic venant de ces deux villes.

En l'absence de données de stations météo-routières nous utiliserons celles des stations météorologiques respectivement de Lyon et de Chambéry selon les cas (la distance entre les stations météorologiques et routières éloignées est dans chaque cas d'environ $10 \mathrm{~km}$ ). Ces données présentent néanmoins de gros inconvénients : elles sont uniquement qualitatives (pluie ou non, neige ou non...) et agrégées sur l'heure. On ne peut ainsi pas différencier un créneau horaire avec de fortes pluies d'un créneau avec juste quelques gouttes observées.

\section{La route $n^{\circ} 3$ de la $M E X$}

Les données recueillies sur le réseau MEX (Tokyo metropolitan expressway) sont un peu plus complètes. Ce réseau compte actuellement 24 voies rapides payantes et dessert l'aire métropolitaine de Tokyo (voir Figure 4). Nous nous intéressons principalement à la route $n^{\circ} 3$ (Shibuya Route), une $2 \times 2$ voies qui relie la « jonction Tanimachi » à la «jonction Yoga » (jonctions avec d'autres routes non intégrées à la MEX).

Les données disponibles sont des débits pour chaque intervalle de 5 minutes sur la station $\mathrm{n}^{\circ} 554$ de la route $\mathrm{n}^{\circ} 3$ pour la période du $1^{\text {er }}$ août 1999 au 6 avril 2000 seulement, dans le sens Yoga vers Tanimachi. Des données météorologiques relatives à l'intensité des précipitations (pluie uniquement) agrégées sur l'heure sont également disponibles. On dispose également des heures approximatives de début et de fin des accidents survenant sur la route $\mathrm{n}^{\circ} 3$.

\section{Fonctionnement des sections}

Il s'agit dans la suite d'identifier uniquement les impacts de la météorologie défavorable sur le trafic. Il est d'abord nécessaire, comme nous l'avons dit en préambule de cette partie, d'isoler les sources de variation de la demande, et donc de bien comprendre le fonctionnement de l'infrastructure. Pour ce faire, nous avons examiné les profils moyens (débit horaire en fonction de l'heure de la 


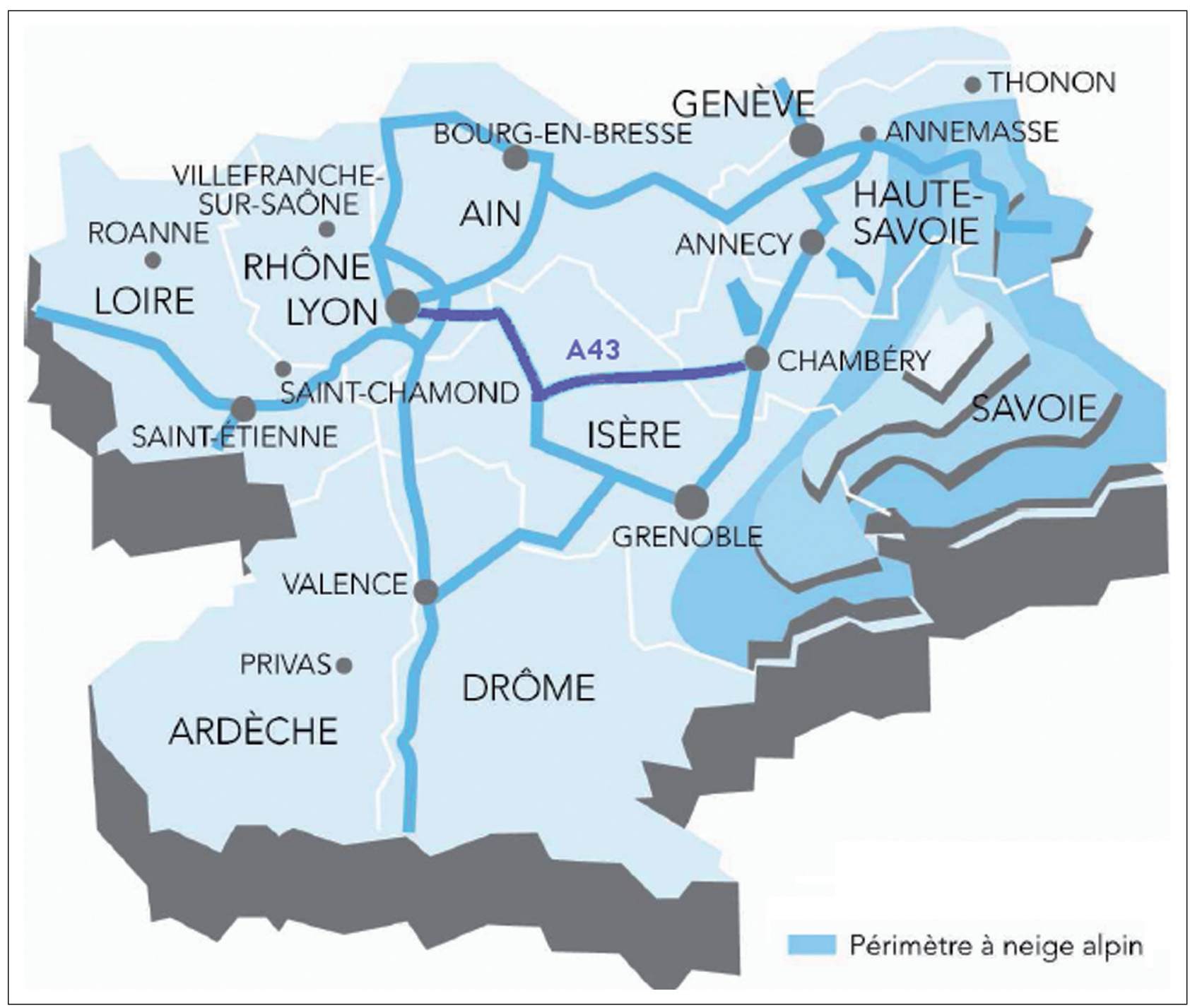

Fig. 3 Localisation de l'axe autoroutier Lyon-Chambéry vis-à-vis du secteur alpin (adapté de l'observatoire régional du tourisme RhôneAlpes)

journée) des différents jours de la semaine aux points de mesure disponibles.

Dans la partie suivante, dans un souci de représentativité statistique, nous réalisons des regroupements de jours aux profils similaires (à travers les mois et les années) afin de pouvoir comparer, d'un côté une base de jours dits « secs » (sans événements météorologiques majeurs) avec des jours dits « pluvieux » ou « neigeux » dans le cas des données de l'A43.

Dans les deux cas, et malgré le caractère périurbain du premier jeu de données, on observe des profils réguliers les jours de semaine, avec des heures de pointe le matin et le soir. Par contre, les trajets des week-ends sont trop irréguliers pour pouvoir effectuer de quelconques regroupements. La cause de ce phénomène est certainement la forte proportion de déplacements effectués dans le cadre d'un motif « loisirs » et qui ne sont donc pas forcément les mêmes d'un dimanche à l'autre par exemple. Dans le cas de la section de l'A43, la demande des vendredis soirs est également trop variable d'un vendredi sur l'autre pour effectuer des regroupements. Cela s'explique par l'existence de déplacements depuis Lyon vers les pistes de ski durant la période hivernale.

\section{Étude de cas en milieu interurbain : la section Lyon-Chambéry de l'A43}

\section{Constitution des échantillons}

Il s'agit maintenant dans cette partie de former des échantillons (par exemple les demandes des jours secs d'un 


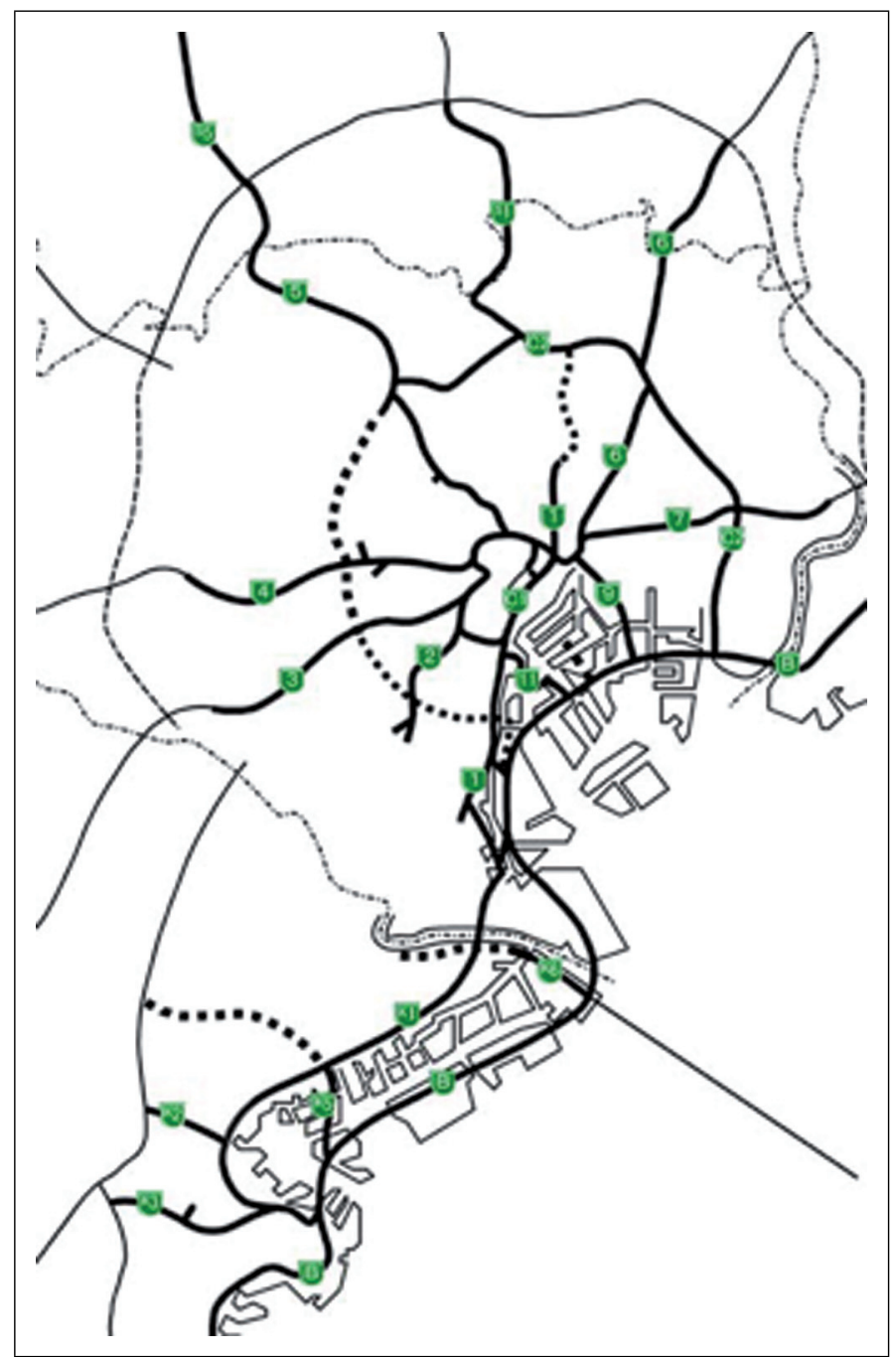

Fig. 4 La route $n^{\circ} 3$ et le réseau autoroutier de Tokyo [28]

côté et les demandes des jours pluvieux de l'autre), et ensuite de les comparer grâce à des méthodes de statistique descriptive. Nous étudions l'impact des événements neigeux et des événements pluvieux sur les volumes de trafic (qui peuvent être interprétés comme la demande en dehors des périodes de congestion) pour certains jours ou groupes de jours de la semaine.

Dans le cas de la section A43, nous allons comparer séparément les demandes des lundis, puis des mardis, puis des mercredis et enfin des jeudis. Les jours de vacances scolaires et fériés sont exclus, car atypiques d'un point de vue de la demande. Nous nous intéressons plus particulièrement aux périodes importantes du point de vue de l'exploitation routière : les heures de pointe du matin (période $6 \mathrm{~h}-11 \mathrm{~h})$ et du soir $(15 \mathrm{~h}-21 \mathrm{~h})$, mais aussi la période creuse de la mi-journée (11 h-15 h). On encadre de façon assez large les véritables périodes d'heures de pointe pour s'assurer de ne pas biaiser l'étude par des phénomènes d'étalement de l'heure de pointe parfois observés (dus à des réductions de vitesse, des régulations...), et bien aller au-delà de la fin de la congestion, pour mesurer correctement la demande. L'agrégation horaire des données permet certainement d'écarter ce phénomène.

Nous évoquons plus tôt dans cet article le manque de données concernant l'état de la chaussée. Cela peut poser problème dans le cas de la définition des jours secs, car on se base uniquement sur les précipitations qui surviennent durant la période. Or il est possible, par exemple, qu'il ait neigé avant le début de l'heure de pointe et que par conséquent il se soit formé une couche de neige au sol qui perdure pendant l'heure de pointe du matin, voire au-delà. Nous avons donc créé un indicateur pour les jours secs qui permet de dire s'il a neigé ou plu durant les $5 \mathrm{~h}$ précédant la période considérée (HPM, HPS ou heures creuses). Le critère de $5 \mathrm{~h}$ a été défini assez arbitrairement, mais on peut néanmoins supposer que cela permet en général soit 
à la chaussée de sécher dans le cas de la pluie ou bien de déneiger en cas de neige. Le même indicateur est utilisé pour étudier les données japonaises.

Nous allons maintenant comparer nos échantillons à l'aide de plusieurs tests statistiques paramétriques ou non-paramétriques.

\section{Comparaison des intervalles de confiance des moyennes}

Une première méthode assez simple consiste à calculer les moyennes de chaque échantillon, les intervalles de confiance correspondants, puis de comparer ces intervalles. Pour chaque échantillon de débits journaliers «secs » et de débits journaliers «pluvieux », nous choisissons de calculer les intervalles de confiance à $95 \%$ (ou au seuil de risque de $5 \%$ ) de la moyenne.

On sépare l'étude de la demande de trafic totale et l'étude de la demande de trafic poids-lourd uniquement.

On observe tout d'abord qu'il est impossible de conclure dans tous les cas, et ce quel que soit le type de trafic considéré sur un potentiel effet de la pluie ou de la neige sur l'heure de pointe du soir. Cela peut sans doute s'expliquer par le fait qu'à l'HPS, les trajets de retour « domicile-travail » doivent obligatoirement être réalisés, alors que sur le matin des changements sont encore envisageables.

Les intervalles de confiance ne s'intersectent pas dans plusieurs cas à l'heure de pointe du matin et pendant les heures creuses : dans ces cas l'on peut dire qu'il y a un effet de la pluie, mais aussi de la neige sur les volumes de trafic à l'heure de pointe du matin. La validation de cette hypothèse n'est par contre pas systématique, même si on rappelle qu'une intersection des intervalles ne traduit pas forcément une absence d'effet, mais entraîne juste une impossibilité de conclure.

Pour le trafic total uniquement, la pluie et la neige ont, lorsque l'effet est avéré, une action réductrice sur le volume de trafic de la période considérée. Ceci n'est cependant pas vrai dans le cas du trafic PL.

On observe également que les tests se révèlent plus souvent positifs pour les trafics de l'heure de pointe du matin que pour les autres. Cela ne veut pas dire pour autant que la réduction est la plus importante à l'heure de pointe dans le cas où elle existe. La répartition des cas positifs permet d'envisager une importance plus forte de la neige que de la pluie sur les volumes de trafic VLPL de l'HPM. La répartition de ces cas pousse également à faire l'hypothèse d'une plus forte influence sur le trafic VLPL que sur le trafic VL (ce qui serait conforme à la littérature).

On voit donc que même si les conclusions relatives à l'utilisation de ce test sont relativement difficiles à établire (d'autant que nous ne maîtrisons absolument pas l'intensité du phénomène), le test des intervalles de confiance des moyennes permet d'entrevoir un effet de la neige et de la pluie sur le trafic, notamment à l'heure de pointe du matin, mais a priori surtout sur le trafic VLPL. Une méthode de statistique descriptive basée sur des représentations graphiques va nous permettre d'affiner nos analyses.

\section{Utilisation de diagrammes en boûte}

Nous allons étudier les liens entre une variable quantitative dépendante, le volume de trafic (que ce soit sur l'HPM, l'HPS ou les heures creuses), et une variable qualitative indépendante, à savoir la variable météo (avec trois modalités, «sec », « pluie » ou « neige »). Les diagrammes en boîte fournissent une illustration très parlante de ces liens en comparant sur un même graphique la médiane, les quartiles et les déciles d'une des modalités de la variable indépendante (la météo). Nous faisons également figurer la moyenne sur nos graphiques, même si historiquement elle ne figure pas sur un graphique à moustache classique. Un exemple de graphiques réalisés est disponible en Figure 5 .

Dans le cas général, on retrouve une forme qu'on pourrait qualifier «d'escalier » avec la boîte correspondante à l'échantillon sec plus haute que les autres, la boîte de l'échantillon pluvieux en position intermédiaire et la boîte de l'échantillon neigeux la plus en dessous possible. Il y aurait donc bien un effet de la pluie et de la neige sur les volumes de trafic VLPL (et donc la demande) des heures de pointe en semaine et, de plus, l'effet de la neige serait plus important que celui de la pluie. La représentation des quartiles et des déciles permet également de voir la grande variabilité due à la petite taille des échantillons. La comparaison des médianes des différents échantillons permet également de se rendre compte que les variations sont pour l'HPM et l'HPS en général faibles, avec toujours une différence plus importante dans le cas des échantillons neigeux. Cette analyse est valable pour les deux sens de circulation. Rappelons également que précédemment il n'avait pas été possible de conclure sur un effet potentiel à l'heure de pointe du soir, alors qu'ici un effet semble exister.

Les résultats pour les heures creuses sont plus compliqués à interpréter : si les graphiques du sens Lyon-Chambéry inciteraient à généraliser l'analyse des HPM et des HPS aux cas des heures creuses, il n'en est rien pour ceux $\mathrm{du}$ sens inverse qui amène à relativiser les effets de la pluie surtout. Ceux de la neige restent néanmoins visibles.

Le cas du trafic PL est plus complexe. Dans le sens LyonChambéry, l'allure en « escalier » évoquée précédemment se retrouve difficilement. On s'aperçoit d'abord en observant 


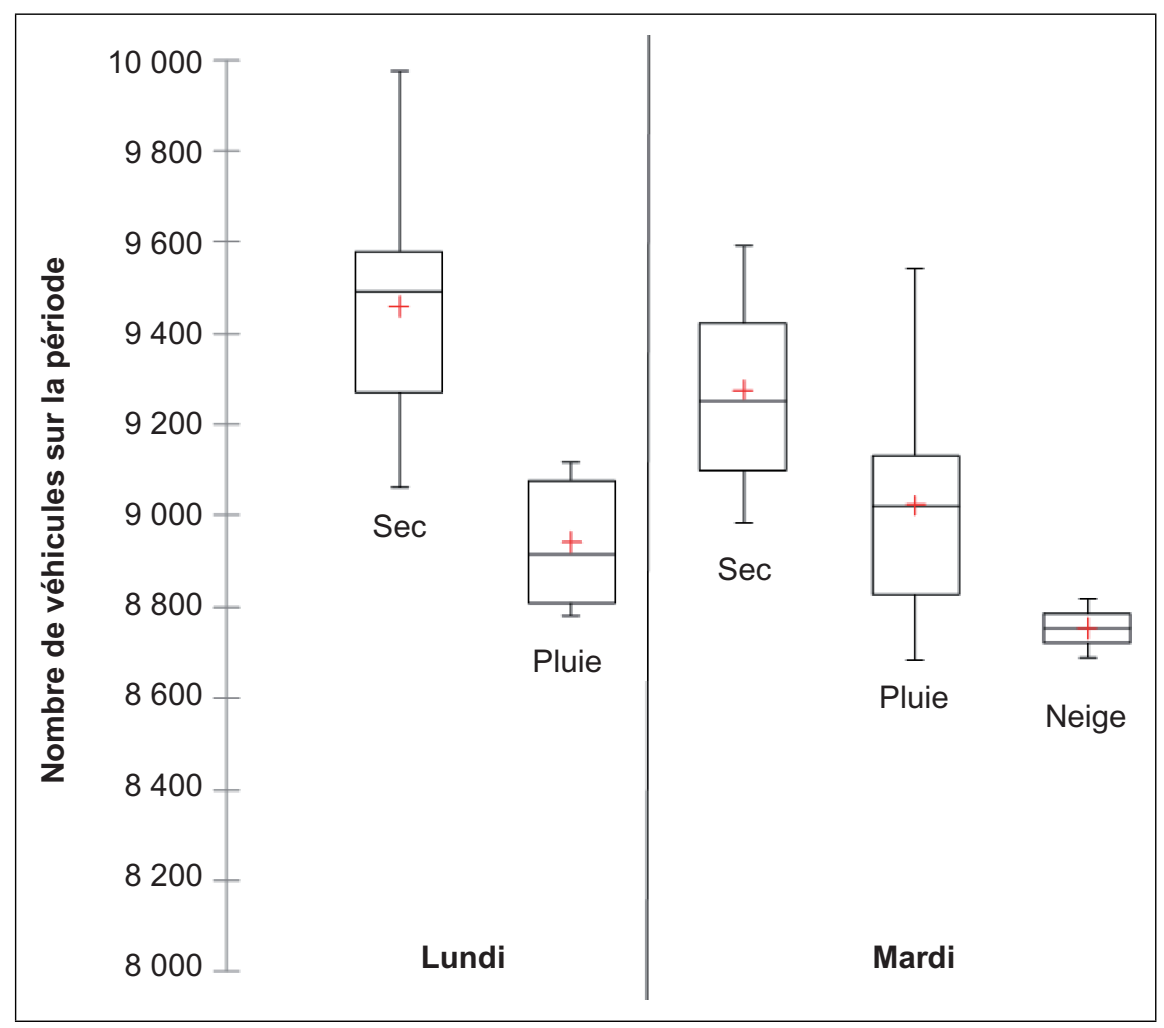

Fig. 5 Exemple de diagrammes en boîte pour les heures de pointe du matin (trafic total) sur la station PK 21 (sens Lyon-Chambéry)

les échantillons secs que le trafic PL est vraiment faible et assez variable (voir les premiers et derniers déciles des jours secs), ce qui rend de fait l'interprétation compliquée. Quelle que soit la période (HPM, HPS ou heures creuses), des réductions de trafic peuvent être observées dans certains cas, mais des augmentations dans d'autres. On peut envisager là une sensibilité plus faible des conducteurs PL aux conditions météorologiques que celles des autres usagers, ce qui aboutirait dans certain cas à ne pas observer de tendance à la baisse alors que dans le cas général si. Une maîtrise de l'intensité du phénomène météorologique permettrait de vérifier cette hypothèse, puisque cela permettrait d'identifier le niveau moyen de neige tombé, par exemple pour chaque échantillon.

Dans le sens inverse, nous sommes confrontés aux mêmes conclusions pour le trafic PL : dans certains cas, il semblerait même qu'en cas de pluie les volumes de trafic PL soient plus importants. Cette fois-ci pourtant le trafic PL moyen est non négligeable comparé à l'autre sens. On pourrait associer cela à des trajets plus fortement contraints. L'existence d'un impact de la neige semble par contre plus visible qu'en sens inverse quelle que soit la période.

On constate bien ici l'avantage de la méthode des diagrammes en boîte qui permet de constater directement les différences entre les échantillons et de conclure qu'il y aurait bien un effet de la neige et de la pluie notamment durant les heures de pointe du matin et du soir pour le trafic général. De plus, cette méthode ne se base en aucune façon sur la nature des distorsions des échantillons (gaussienne ou non). Un autre avantage de cette méthode est qu'elle permet de visualiser directement les problèmes liés à la taille des échantillons.

\section{Application du test de Mann-Whitney et du test d'analyse de la variance}

Le test de Mann-Whitney est un test non paramétrique de comparaison de deux échantillons indépendants, et s'applique essentiellement aux variables numériques. Ce test permet de contrôler l'homogénéité de deux populations, c'est-à-dire de savoir si la distribution des deux populations est identique. Les hypothèses de ce test sont :

- $\mathrm{H}_{0}$ : les échantillons proviennent de la même population (hypothèse nulle) ;

- $\mathrm{H}_{1}$ : les deux échantillons proviennent de deux populations différentes.

Nous avons appliqué le test de Mann-Whitney à chaque couple d'échantillons (sec-pluie du lundi, sec-neige du lundi...) avec un risque de $5 \%$. Si la p-value calculée pour 
Tableau 1 Synthèse des cas où l'on peut rejeter l'hypothèse nulle avec un risque de $5 \%$ (test de Mann-Whitney)

\begin{tabular}{lllll}
\hline & & HPM & HPS & HC \\
\hline \multirow{2}{*}{ VLPL } & Pluie & 2 cas sur 7 & 2 cas sur 8 & 1 cas sur 8 \\
& Neige & 5 cas sur 6 & 1 cas sur 5 & 1 cas sur 4 \\
PL & Pluie & 2 cas sur 7 & 0 cas sur 8 & 1 cas sur 8 \\
& Neige & 1 cas sur 6 & 0 cas sur 5 & 0 cas sur 4
\end{tabular}

chacun des tests est inférieure au niveau de signification retenue ( $5 \%$ ), on doit rejeter l'hypothèse nulle $\mathrm{H}_{0}$. Il faut noter que si la p-value est supérieure à $5 \%$, elle conduit au non-rejet de l'hypothèse nulle.

Le Tableau 1. synthétise l'ensemble des résultats positifs (rejet de l'hypothèse nulle au risque de $5 \%$ ) des tests de Mann-Whitney réalisés. Comme lors de l'analyse des résultats de la comparaison des intervalles de confiance des moyennes des différents échantillons, on trouve la majorité des résultats positifs dans le cas de l'HPM. Ainsi, la majorité des échantillons neigeux et secs de semaine proviendrait de populations différentes, il y aurait donc bien un effet de la neige sur le trafic VLPL en semaine à l'heure de pointe du matin. Les volumes de trafic routier des HPM des jours secs et ceux des jours pluvieux proviennent dans 2 cas sur 7 seulement de populations différentes. Cela est certes faible, mais montre qu'il y a là aussi potentiellement un changement dans les volumes de trafic sous certaines conditions météorologiques, avec a priori un effet plus fort pour la neige que pour la pluie.

Il est par contre à nouveau difficile de tirer des conclusions concernant d'éventuelles modifications sur le trafic PL dans les deux sens de circulation, mais il faut noter néanmoins une élimination de l'hypothèse nulle dans certains cas. Le test de Mann-Whitney ouvre également la porte à un effet sur le trafic VLPL à l'heure de pointe du soir, puisqu'il existe des cas positifs pour la neige et la pluie, et donc où les volumes de trafic sont différents.

À titre indicatif, une analyse de la variance (Anova) a également été effectuée sur chacune des paires d'échantillon (l'hypothèse nulle de ce test est que les moyennes des deux échantillons sont égales). Les tendances sont donc les mêmes que celles évoquées précédemment, avec néanmoins plus de cas positifs pour l'HPS. Ces nouveaux cas positifs amènent à considérer un impact de la neige sur les trafics de cette période (VLPL ou PL), un impact potentiel de la pluie, mais à rejeter tout impact de la pluie sur le trafic PL.

\section{Étude de cas en milieu urbain : la route $n^{\circ} 3$ du réseau autoroutier de Tokyo}

Compte tenu des limites que présentent les données recueillies sur l'A43, nous allons maintenant étudier selon les mêmes méthodes des données récupérées en milieu urbain. Le second intérêt porte sur les données météorologiques : ce ne sont plus les conditions qui sont données mais l'intensité horaire des précipitations. L'intensité des précipitations neigeuses n'est par contre pas fournie. Compte tenu du fonctionnement de la section, nous étudierons les volumes de trafic des mardis, mercredis et jeudis (hors vacances scolaires et jours fériés) ensemble, ce qui permet d'avoir un volume de données suffisant dans chacun des échantillons secs et pluvieux.

\section{Diagrammes en boîte}

Nous allons dans la suite utiliser les mêmes méthodes statistiques que pour le premier jeu de données. Nous avons représenté sur la Figure 6 plusieurs échantillons de jours, suivant l'intensité des précipitations. On représente ici les demandes des jours secs $(0 \mathrm{~mm} / 24 \mathrm{~h})$, les jours où il $\mathrm{y}$ a eu au moins $1 \mathrm{~mm}$ de pluie, $5 \mathrm{~mm}$ de pluie, $10 \mathrm{~mm}$ et $20 \mathrm{~mm}$. On voit d'abord avec l'allure en «escalier» $\mathrm{du}$ diagramme que la pluie aurait une influence sur le nombre de déplacements effectués dans la journée sur l'axe. De plus, la logique et la littérature semblent respectées puisque plus l'intensité des précipitations sur la journée est importante, et plus la réduction de la demande sur la journée le serait corrélativement. Les médianes et les moyennes sont à chaque fois plus faibles avec l'augmentation des précipitations (décroissance), de même que les «boîtes » contenant une grande partie des valeurs.

Nous avons également réalisé la même opération pour la période clé de l'heure de pointe du matin (6h-10h), mais aussi pour la journée $(6 \mathrm{~h}-21 \mathrm{~h})$. On retrouve dans les deux cas les conclusions que nous venons de faire pour l'ensemble de la journée.

\section{Tests statistiques :}

\section{ANOVA, Mann-Whitney et intervalles de confiance}

Pour compléter les études précédemment faites, des analyses statistiques ont été menées sur les données trafics et temps de parcours. Dans le Tableau 2 nous avons rassemblé les résultats des tests de superposition des intervalles de confiance, d'analyse de la variance (Anova, paramétrique) et de Mann-Whitney (non paramétrique) pour des échantillons de demande journalière selon différents 


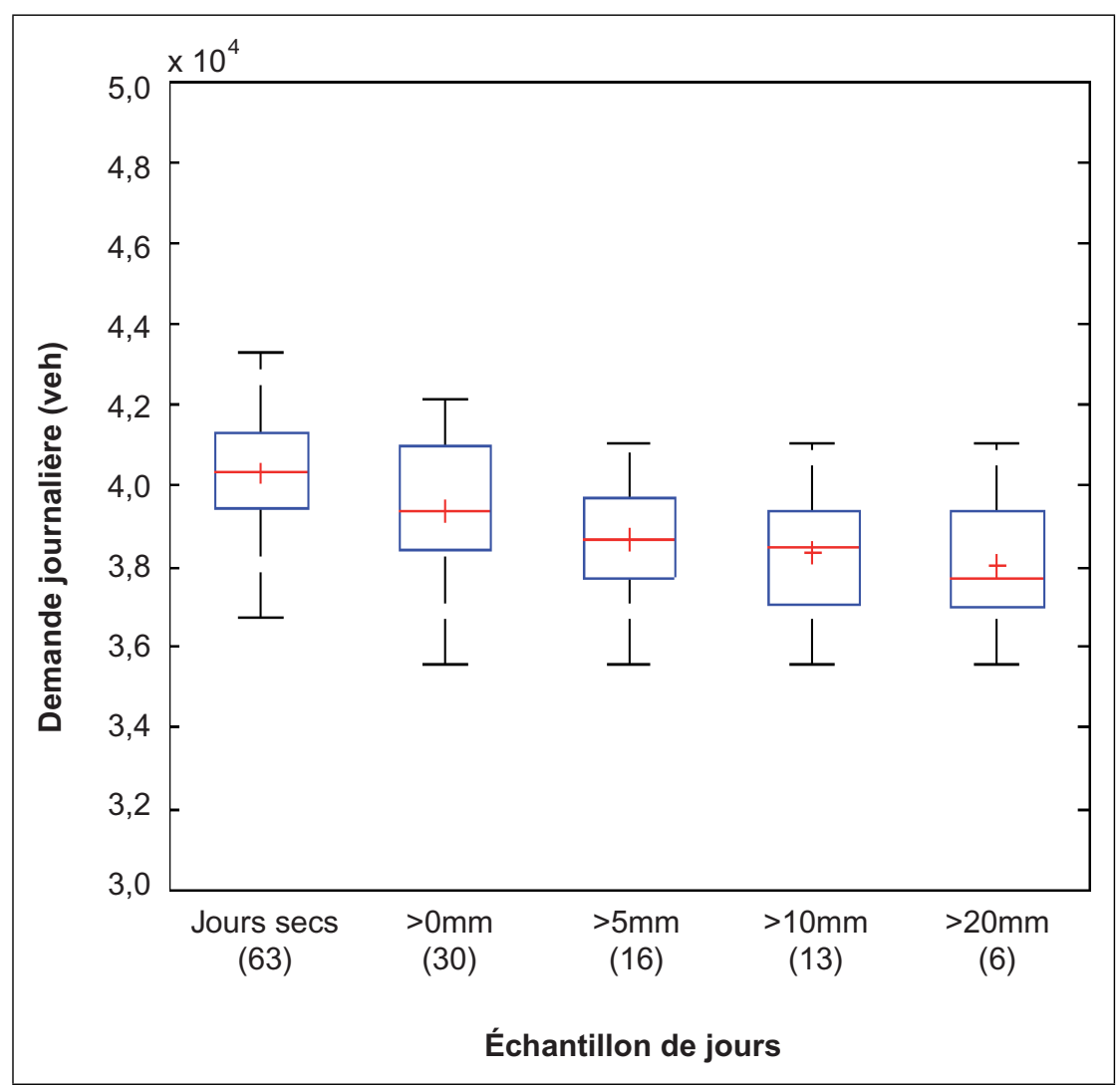

Fig. 6 Diagrammes en boîte des demandes journalières des mardis, mercredis et jeudis normaux

\begin{tabular}{|c|c|c|c|c|c|c|c|c|c|c|c|c|}
\hline & Jours secs & $>$ & $0 \mathrm{~mm}$ & $>$ & $2 \mathrm{~mm}$ & $>$ & $4 \mathrm{~mm}$ & $>$ & $6 \mathrm{~mm}$ & $>8$ & $8 \mathrm{~mm}$ & $>10 \mathrm{~mm}$ \\
\hline Taille (nb de jours) & 63 & & 30 & & 21 & & 17 & & 15 & & 15 & 13 \\
\hline Moyenne (veh/jour) & 40296 & & 39375 & & 39010 & & 38628 & & 38586 & & 38586 & 38350 \\
\hline IC 95 \% (borne inf.) & 39896 & & 38745 & & 38257 & & 37847 & & 37722 & & 37722 & 37436 \\
\hline IC $95 \%$ (borne sup.) & 40697 & & 40005 & & 39764 & & 39409 & & 39451 & & 39451 & 39264 \\
\hline Superposition des IC & - & & Oui & & Non & & Non & & Non & & Non & Non \\
\hline Anova & - & & $1,46 \%$ & & $0,28 \%$ & & $0,03 \%$ & & $0,05 \%$ & & $0,05 \%$ & $0,02 \%$ \\
\hline Mann Whitney & - & & $2,33 \%$ & & $0,39 \%$ & & $0,05 \%$ & & $0,08 \%$ & & $0,08 \%$ & $0,04 \%$ \\
\hline
\end{tabular}

critères météorologiques. À chaque fois le test est effectué en comparant l'échantillon pluvieux à l'échantillon sec. Les mêmes analyses ont également été conduites pour la demande de l'HPM et celle de la journée (6 h-20 h).

Concernant la demande journalière, on note qu'il n'y a pas superposition de l'intervalle de confiance de la moyenne de la demande des jours secs et celle de chacun des jours pluvieux, à l'exception de celle des jours où il a plu moins de $1 \mathrm{~mm}$ en 24h. On peut donc dire que la pluie a bien un effet (négatif) sur les volumes de trafics journaliers, à partir du moment où il pleut plus de $1 \mathrm{~mm}$ en $24 \mathrm{~h}$.
Par contre, si on compare les intervalles de confiance des moyennes de la demande de chacun des jours pluvieux, il y a à chaque fois superposition. On ne peut donc pas affirmer a priori que l'intensité des précipitations a une influence statistiquement significative sur la valeur du débit journalier. L'influence de l'intensité des précipitations sur les débits est en fait difficile à approcher puisqu'à cause de notre critère de définition des jours pluvieux certains jours sont dans plusieurs échantillons. Or plus on élève les critères, et plus les échantillons se réduisent, et donc moins il y a de différences entre ceux-ci. La solution serait, à l'instar des jours secs, de retenir seulement les jours où il $\mathrm{y}$ a eu 
une quantité finie de pluie tombée en 24 h. Néanmoins, compte tenu du jeu de données, la taille des échantillons serait malheureusement trop petite.

On parvient aux mêmes conclusions concernant les autres périodes de ce test, même si dans le cas de la période $6 \mathrm{~h}-20 \mathrm{~h}$ la pluie a une influence sur les débits dès $1 \mathrm{~mm}$. Les mêmes remarques peuvent être faites quant à un effet négatif de la pluie sur les temps de parcours quels que soient la période retenue et le niveau de précipitations.

Concernant le test d'analyse de la variance, que l'on s'intéresse à la journée entière, à l'heure de pointe du matin ou à la période $6 \mathrm{~h}-21 \mathrm{~h}$, les p-values des Anova amènent à rejeter l'hypothèse nulle. La moyenne de la demande les jours secs (respectivement sur une des deux autres périodes) est donc différente de la moyenne des jours pluvieux (idem pour les autres périodes), et ceci quel que soit le critère météorologique retenu. Notons que le test de Mann-Whitney, qui est l'équivalent non paramétrique de l'Anova, amène dans tous les cas à rejeter l'hypothèse nulle.

Après avoir également mis en évidence une influence de la météorologie sur la demande de trafic en milieu urbain, nous allons maintenant essayer dans la partie suivante d'estimer les réductions de demande de trafic à partir de l'ensemble de nos données en établissant différents modèles de prévision de volume de trafic journalier.

\section{Modélisation de l'influence de la météorologie sur la demande}

Nous venons de voir qu'il n'est souvent pas possible de rejeter un effet de la météorologie (pluie ou neige) sur le trafic. À partir des données horaires récoltées sur la période 2008-2010 sur 1'A43 uniquement (les études réalisées à partir des données issues de la MEX se révélant moins concluantes, voir en fin de partie), nous allons réaliser un certain nombre de régressions multiples afin de quantifier l'effet de la météorologie sur les volumes journaliers. Le but est de réaliser des modèles de prévision de trafic à partir de différentes variables explicatives.

\section{Préalables méthodologiques}

\section{Utilisation de la régression linéaire multiple}

Nous reprennons la démarche utilisée par Keay et Simmonds sur des données de l'agglomération de Melbourne sur la période 1989-1996. Keay et Simmonds indiquent que les modèles de régression sont adaptés à l'analyse des débits journaliers. Les modèles de régression linéaire multiple que nous allons mettre en place dans la suite vont chercher à expliquer les volumes moyens de trafic journaliers sur 1'A43 (variable dépendante) en fonction de divers paramètres temporels et bien sûr de facteurs météo (variables indépendantes). Knapp et al. et Datla et Sharma ont également utilisé cette approche afin d'étudier les effets de la neige sur les volumes de trafic journalier [3] [29] [31].

On rappelle que la régression linéaire multiple est une généralisation de la régression linéaire simple à plusieurs variables explicatives, et consiste à déterminer l'estimation des paramètres du modèle (coefficients, variant de 1 à $\mathrm{p}$ avec $\mathrm{p}$ le nombre de variables explicatives) et à estimer la validité de la relation entre la variable indépendante (avec i variant de 1 à $n, n$ étant le nombre d'observations) et les variables indépendantes ( $\mathrm{i}$ variant de 1 à $\mathrm{n}, \mathrm{k}$ variant de 1 à $\mathrm{p}$ ). Cette relation peut s'écrire sous cette forme :

$$
Y_{i}=a_{0}+a_{1} X_{i 1}+a_{2} X_{i 2}+\ldots+a_{p} X_{i p}
$$

\section{Expression du modèle de prévision du trafic journalier moyen}

Dans le cas de la prévision de volumes de trafic journaliers, la forme générale des modèles que nous allons mettre en œuvre est :

$$
\begin{aligned}
V=a+b_{1} \text { Lundi } & +b_{2} \text { Mardi }+\ldots+b_{6} \text { Samedi } \\
& +c_{1} \text { Janvier }+\ldots+c_{11} \text { Novembre } \\
& +d_{1} \text { Année } 09+d_{2} \text { Année } 10+\mathrm{e}_{1} \text { Férié } \\
& +e_{z} \text { Vacance }+f_{1} \text { Météo } 1+f_{\mathrm{z}} \text { Météo } 2 \\
& +\ldots
\end{aligned}
$$

Ce ne sont pas forcément exactement avec toutes ces variables explicatives que le modèle s'écrira dans la suite, mais cette forme générale sert de cache pour expliquer le fonctionnement du modèle. Dans cette expression, $a, b_{1-6}, c_{1-11}, d_{1}, d_{2}, e_{1}, e_{2}, f_{1-m}$ (m désigne le nombre de variables météo introduites) sont les coefficients du modèle. $\mathrm{V}$ désigne le volume de trafic journalier, Lundi-Mardi...-Samedi sont des variables binaires relatives au jour de la semaine, Janvier-. . .-Novembre des variables binaires relatives au mois, Année09 et Année10 celles relatives à l'année, Férié la variable binaire indiquant les jours fériés, et Vacance celle renvoyant aux jours de vacances scolaires. Les variables relatives aux conditions météorologiques (Météo1, Météo2...) sont des variables binaires ou quantitatives. On peut par exemple utiliser la quantité de neige tombée (quantitative), la température moyenne (quantitative) ou bien s'il a plu durant la journée ou non (binaire). 
Il est à noter que les variables « dimanche », « décembre » et « 2008 » ne figurent pas dans l'équation. L'introduction de ces variables n'est pas nécessaire en raison de la relation linéaire qui existe entre chacune de ces variables et sa catégorie ou classe (respectivement les autres variables binaires du jour de la semaine, du mois et de l'année). La variable « dimanche » correspond forcément au cas où les variables Lundi-Mardi-....-Samedi prennent toutes la valeur 0 , c'est notre référence. C'est pour cette raison que le terme constant contient en fait l'effet du dimanche. Par voie de conséquence, le terme représente la différence entre le trafic moyen du lundi et celui du dimanche. Par extension, le terme contient aussi l'effet du mois de décembre, de l'année 2008... et plus généralement de toutes les variables utilisées comme situation de référence.

Afin de simplifier au maximum l'interprétation du modèle, nous allons comme Keay et Simmonds normaliser la série des volumes journaliers afin d'avoir un volume journalier moyen égal à 100. Cela signifie que dans nos modèles la plupart des coefficients des paramètres seront exprimés directement en pourcentage de variations par rapport à la moyenne de la série des volumes (puisqu'un coefficient de -10 indique par exemple une diminution de 10 par rapport à 100 , donc de $10 \%$ ) [29].

\section{Expression du modèle de prévision du trafic journalier moyen}

Pour la suite, nous avons créé plusieurs variables binaires pour la classe des jours de la semaine, des mois de l'année et de l'année notamment. Des variables furent également nécessaires pour modéliser l'effet des jours fériés et des vacances scolaires (de la zone A, zone de Lyon). Nous verrons que dans certains cas des raffinements ont été introduits en créant une variable binaire pour chaque type de vacances (vacances de noël, d'hiver, de printemps, d'été et de la Toussaint), ceci afin d'améliorer la qualité globale du modèle.

Nous allons réaliser plusieurs modèles de prévision du trafic VLPL (véhicules légers et poids lourd, donc le trafic total) : un pour les jours de semaine (du lundi au jeudi), un pour le vendredi, et un autre pour le week-end. Enfin, un modèle de prévision du trafic PL moyen journalier pour l'intégralité de la semaine est entrepris. Nous voyons les raisons de ces distinctions dans la suite. La méthodologie est identique à chaque fois. Nous réalisons un modèle de prévision de trafic dit « de base », c'est-à-dire sans les variables météorologiques. Puis nous introduirons chacune de ces variables une par une et séparément. Cela nous permet d'estimer à chaque fois l'apport de la variable météo utilisée, et bien sûr le coefficient du modèle allant avec cette variable.
Les variables météo successivement introduites sont en général basées sur l'occurrence du phénomène sur la journée (par exemple le nombre d'heures où la pluie a été détectée durant la journée).

Précisons que nous n'utilisons que les données de la station PK21 dans le sens Lyon-Chambéry (station PK21S). Nous utilisons néanmoins cette fois-ci l'intégralité des données, c'est-à-dire les données journalières sur l'intégralité des années 2008, 2009 et des premiers mois de 2010 (soit 867 échantillons, après retrait des jours où il manquait des données sur certaines heures et où il était donc impossible de calculer un trafic journalier). Enfin, rappelons pour mémoire que la définition des heures de pointe utilisée est pour l'HPM de $6 \mathrm{~h}$ à $11 \mathrm{~h}$, pour les heures creuses de 11 $\mathrm{h}$ à $15 \mathrm{~h}$, et pour les HPS de $15 \mathrm{~h}$ à $21 \mathrm{~h}$.

\section{Étude du modèle expliquant le trafic moyen journalier de la semaine}

\section{Construction du modèle}

Conformément à la méthodologie exposée, nous allons d'abord construire le modèle de base, c'est-à-dire sans introduire des variables météo. Il s'agit à cette étape de définir quel est le modèle de régression le plus adapté.

Nous souhaitions au départ concevoir un modèle de régression capable d'expliquer le trafic journalier moyen total (véhicules légers et poids-lourds) pour l'ensemble des jours de la semaine. Malheureusement, il fut impossible d'obtenir un modèle avec un coefficient de détermination multiple $\mathrm{R}^{2}$ supérieur à 0,36 , ce qui reste assez faible. Ce résultat peut sans doute s'expliquer par la trop grande variabilité du trafic qui existe entre ces jours. La séparation en trois modèles distincts (un du lundi au jeudi, un pour le vendredi et un pour le week-end) permet de contourner cette difficulté.

Nous ne retenons donc pour ce premier modèle que les données des lundis, mardis, mercredis et jeudis (soit 494 échantillons). Nous réalisons au départ plusieurs essais afin d'avoir le « meilleur » modèle possible (c'est-à-dire avec le meilleur $\mathrm{R}^{2}$ ). L'ajout de chaque classe de variables (d'abord, les jours de la semaine, les jours fériés et les vacances, les mois, et enfin les années) conduit systématiquement à une amélioration du $\mathrm{R}^{2}$ pour atteindre finalement $66,5 \%$. À titre de comparaison, la qualité des modèles de régression de Keay et Simmonds atteignait les $90 \%$ (structure du modèle identique, mais remplacement de l'ensemble des variables binaires du mois et de l'année par une seule variable qualifiée «d'index temporel » qui vaut 1 pour le premier jour de la plage des données considérées, 2 pour le second. . . Cette méthode ne conduit pas dans notre cas à des résultats probants). Cette différence s'explique certainement par la nature des données prises en compte dans leur 
étude : la plage temporelle est beaucoup plus grande ( 7 ans de données) et de nombreuses routes de l'agglomération sont prises en compte, et c'est essentiellement un milieu urbain très dense qui est étudié, donc un trafic beaucoup plus régulier sur l'année [29].

Les valeurs des coefficients du modèle et de leurs intervalles de confiance sont notamment disponibles dans le Tableau 3. Dans ce modèle, les variables « Lundi », «Janvier» et « 2008 » sont prises comme références de leurs classes respectives. La colonne « Sig. » pour significativité contient les p-value du test de Student qui permet de tester la significativité de chacun des coefficients (H0 : le coefficient est nul et $\mathrm{H} 1$ : le coefficient est non-nul). On retient toujours un risque de $5 \%$. On voit ainsi que l'ensemble des variables joue un rôle significatif dans le modèle. Pour les coefficients du mardi et du mercredi, on doit accepter l'hypothèse nulle. Cela peut signifier une absence de corrélation entre ces variables et la variable dépendante (le volume journalier moyen) ou bien une corrélation avec une autre variable explicative. C'est sans doute ce dernier cas qui est à privilégier, le volume journalier moyen du mardi et du mercredi étant sans doute

\begin{tabular}{|c|c|c|c|c|}
\hline & \multirow[t]{2}{*}{ Coef. } & \multirow[t]{2}{*}{ Sig. } & \multicolumn{2}{|l|}{$\begin{array}{l}\text { IC à } 95 \% \\
\text { du } \\
\text { coefficient }\end{array}$} \\
\hline & & & borne inf. & $\begin{array}{l}\text { Borne } \\
\text { sup. }\end{array}$ \\
\hline Constante & 84,086 & 0,000 & 82,045 & 86,127 \\
\hline Mardi & $-1,383$ & 0,072 & $-2,892$ & 0,126 \\
\hline Mercredi & 0,827 & 0,283 & $-0,685$ & 2,339 \\
\hline Jeudi & 6,243 & 0,000 & 4,737 & 7,749 \\
\hline Férié & $-24,739$ & 0,000 & $-27,84$ & $-21,64$ \\
\hline VacToussaint & 6,055 & 0,001 & 2,595 & 9,516 \\
\hline Février & 7,613 & 0,000 & 5,248 & 9,979 \\
\hline Mars & 10,093 & 0,000 & 7,796 & 12,39 \\
\hline Avril & 8,403 & 0,000 & 6,098 & 10,708 \\
\hline Mai & 9,921 & 0,000 & 7,427 & 12,415 \\
\hline Juin & 19,09 & 0,000 & 16,457 & 21,722 \\
\hline Juillet & 22,045 & 0,000 & 19,448 & 24,642 \\
\hline Août & 2,852 & 0,037 & 0,174 & 5,53 \\
\hline Septembre & 14,739 & 0,000 & 12,127 & 17,35 \\
\hline Octobre & 14,067 & 0,000 & 11,319 & 16,814 \\
\hline Novembre & 13,147 & 0,000 & 10,359 & 15,934 \\
\hline Décembre & 17,539 & 0,000 & 14,968 & 20,11 \\
\hline Année 2009 & 6,021 & 0,000 & 4,866 & 7,175 \\
\hline Année 2010 & 9,936 & 0,000 & 8,202 & 11,67 \\
\hline
\end{tabular}

peu différent de celui du lundi (la référence). On pourrait retirer ces variables du modèle, mais elles améliorent tout de même légèrement le $\mathrm{R}^{2}$. Dans toute la suite, les variables dont les coefficients ne sont pas significatifs seront indiquées en italique.

Le coefficient de la variable « vacance » était fortement non significatif (le risque de rejeter l'hypothèse nulle alors qu'elle est vraie était de $96 \%$ ), celle-ci fut donc retirée du modèle. Plusieurs tests furent donc effectués avec des variables par type de vacances scolaires. Seul le coefficient concernant les vacances de la Toussaint fut considéré comme significatif, c'est donc la seule variable de la classe des vacances qui a été conservée dans le modèle. Cette non-significativité provient sans doute d'une corrélation avec certaines variables de mois qui sont en grande partie des mois de vacances.

\section{Interprétation du modèle obtenu (semaine)}

Le modèle établi permet d'étudier les variations de trafics sur l'A43, sans pour l'instant faire intervenir les conditions météorologiques. Outre la faible différence entre les trafics moyens du lundi, mardi, mercredi et même jeudi, on constate bien une augmentation moyenne du trafic journalier entre les années 2008 et 2009 (entre 4,9\% et 7,2 \% si 1'on considère les intervalles de confiance du coefficient) et entre 2008 et 2010 (entre $8,2 \%$ et $11,7 \%$ ). Cette augmentation est bien plus faible entre 2009 et 2010. Rappelons que la valeur retenue dans la partie précédente entre 2009 et 2010 était de $14 \%$, mais celle-ci était calculée uniquement pour le trafic journalier du vendredi alors qu'ici nous sommes dans le cas d'une moyenne sur l'ensemble des jours.

Il est cohérent de trouver une forte réduction pendant les jours fériés $(-21,7 \%$ et $-27,8 \%)$. Le volume de trafic moyen journalier varie de mois en mois, le plus bas étant atteint en janvier, le plus fort en juillet sans doute à cause des départs en vacances et des week-ends de loisirs plus importants avec l'été. L'intégration des mois permet d'ailleurs de s'affranchir en partie des effets saisonniers pour la suite, et de se concentrer sur l'impact propre d'une météorologie défavorable.

Nous réalisons donc ensuite plusieurs modèles, un pour chaque variable météorologique listée précédemment, intégrée au modèle de régression du trafic VLPL de base. Nous retenons à chaque fois plusieurs informations utiles que nous synthétisons en Tableau 4.

La différence entre le $\mathrm{R}^{2}$ du nouveau modèle et celui du modèle de base peut être considérée comme une indication raisonnable de la contribution de la variable considérée au modèle. Par exemple, notre modèle de base a ici un $\mathrm{R}^{2}$ de $66,5 \%$. Si le modèle intégrant la température moyenne a 


\begin{tabular}{|c|c|c|c|c|c|}
\hline $\begin{array}{l}\text { Effets des variables } \\
\text { météo seules }\end{array}$ & Variation du $\mathbf{R}^{2}$ & Coef. & Significativité & Borne inf. IC à $95 \%$ & Borne sup. IC à $95 \%$ \\
\hline Jour Pluvieux & $+0,2 \%$ & $-0,833$ & 0,137 & $-1,931$ & 0,265 \\
\hline Jour Neigeux & $+0,7 \%$ & $-4,109$ & 0,003 & $-6,796$ & $-1,423$ \\
\hline Occurrence pluie $24 \mathrm{~h}$ & $+0,3 \%$ & $-0,156$ & 0,035 & $-0,301$ & $-0,011$ \\
\hline Occ. neige $24 \mathrm{~h}$ & $+1,3 \%$ & $-0,610$ & 0,000 & $-0,883$ & $-0,338$ \\
\hline Occ. pluie HPM & $+0,4 \%$ & $-0,560$ & 0,027 & $-1,055$ & $-0,065$ \\
\hline Occ. pluie HPS & $+0,2 \%$ & $-0,282$ & 0,184 & $-0,699$ & 0,134 \\
\hline Occ. pluie HC & $+0,1 \%$ & $-0,396$ & 0,224 & $-1,035$ & 0,243 \\
\hline Occ. neige HPM & $+0,8 \%$ & $-1,559$ & 0,001 & $-2,460$ & $-0,658$ \\
\hline Occ. neige HPS & $+0,8 \%$ & $-1,679$ & 0,001 & $-2,676$ & $-0,681$ \\
\hline Occ. neige $\mathrm{HC}$ & $+0,6 \%$ & $-1,917$ & 0,004 & $-3,206$ & $-0,629$ \\
\hline Température moy. & $=$ & $-0,006$ & 0,935 & $-0,150$ & 0,138 \\
\hline Température max. & $+0,1 \%$ & 0,035 & 0,565 & $-0,085$ & 0,156 \\
\hline Température min. & $+0,1 \%$ & $-0,073$ & 0,337 & $-0,222$ & 0,076 \\
\hline Vitesse moy. vent & $+0,3 \%$ & $-0,162$ & 0,021 & $-0,299$ & $-0,025$ \\
\hline Vitesse max. vent & $+0,2 \%$ & $-0,062$ & 0,131 & $-0,141$ & 0,018 \\
\hline Vitesse min. vent & $+0,3 \%$ & $-0,224$ & 0,075 & $-0,471$ & 0,023 \\
\hline
\end{tabular}

un $\mathrm{R}^{2}$ de $69,5 \%$, on peut considérer que la température moyenne explique $3 \%$ de la variance du modèle. Cela n'est pas rigoureusement correct car l'ajout d'une variable au modèle entraîne également des modifications sur les autres coefficients, mais des tests entrepris par Keay et Simmonds indiquent que les modifications restent assez faibles [29].

L'augmentation du $\mathrm{R}^{2}$ par rapport au modèle de base (pour les variables dont les coefficients sont significatifs) est comprise seulement entre $0,3 \%$ et $1,3 \%$. On note que les améliorations les plus significatives de la qualité du modèle sont réalisées avec les variables intégrant un effet de la neige (augmentation entre $0,6 \%$ et $1,3 \%$ ). La neige semble donc être le facteur météo contribuant le plus à la variance du modèle. Cela confirme a priori les résultats de la bibliographie, c'est-à-dire que la neige aurait l'impact le plus important sur les volumes de déplacement.

Intéressons-nous maintenant aux valeurs des coefficients du modèle. Si l'impact de la pluie est statistiquement significatif en moyenne les jours de semaine, celui-ci ne l'est pas sur les heures de pointe du soir et aux heures creuses (risque de rejeter $\mathrm{H}_{0}$ alors qu'elle est vraie respectivement de $18,4 \%$ et $22,4 \%$ ). Sur l'ensemble de la journée, la pluie conduirait à une diminution moyenne du trafic journalier moyen de $0,16 \%$ par heure où la pluie est détectée donc avec un intervalle de confiance de $0,01 \%$ à $0,3 \%$. Si la pluie est détectée durant la moitié de la journée, cela ferait donc une réduction moyenne de $1,92 \%$ (avec un intervalle de confiance allant de $0,12 \%$ à $3,6 \%$ ). On peut rapprocher ces valeurs des $1 \%$ à $3 \%$ relevés en moyenne en semaine dans la littérature (et les 1,9\% trouvés pour un jour pluvieux par Keay et Simmonds), même s'il faut rappeler que les mesures sont différentes et se basent en général soit sur un phénomène bien défini (par exemple une journée pluvieuse) soit (pour les modèles) sur l'intensité des précipitations. Les valeurs trouvées sont tout de même assez faibles, mais avec un éventail a priori assez large. L'impact de la pluie semble plus fort sur l'heure de pointe du matin, puisque le coefficient est significatif et plus important que celui trouvé dans le cas de la variable journalière : $-0,56 \%$ en heure de pointe où la pluie est détectée (entre $7 \mathrm{~h}$ et $11 \mathrm{~h}$ ) par rapport au trafic moyen journalier des quatre premiers jours de la semaine (l'intervalle de confiance est là aussi néanmoins important de $-1,1 \%$ à $-0,065 \%$ ).

L'impact de la neige est, comme nous l'avons dit plus haut, beaucoup plus important que celui de la pluie a priori. Le coefficient du modèle correspondant à l'occurrence du nombre d'heures de la journée où la neige est détectée est égal à $-0,61$ (soit $-0,61 \% / \mathrm{h}$ par rapport au volume journalier moyen, sachant que pour la pluie celui-ci était seulement de $-0,16 \% / h)$. Comme prévu, l'impact de la neige est plus important quand elle survient aux heures de pointe, et plus globalement dans la journée puisque le coefficient des heures creuses est significatif et même plus fort que celui de l'HPM et de l'HPS. Si la neige tombe durant l'heure de pointe du matin, la réduction moyenne est de $-1,6 \% / \mathrm{h}$ de l'HPM où il neige ([-2,5\%;-0,66\%]), -1,7\%/h de l'HPS où le phénomène se produit $([-2,7 \% ;-0,7 \%])$ et enfin de 
$-1,9 \% / \mathrm{h}$ s'il neige à la mi-journée $([-3,2 \% ;-0,63 \%])$. Cette valeur plus élevée pourrait signifier que les trajets à motifs non contraints sont bien plus sensibles à une météorologie défavorable que les trajets du type pendulaire.

Concernant l'impact journalier de ces deux phénomènes, l'utilisation des variables « Jour_Pluvieux » et «Jour_Neigeux » permet d'estimer une réduction moyenne du volume moyen de trafic journalier. L'utilisation de ces variables est là encore délicate puisque, d'une part on ne maîtrise l'intensité du phénomène, et d'autre part le critère pour définir ces jours particuliers reste assez large (un jour où il a plu lors d'un seul créneau horaire seulement peut être considéré comme pluvieux). Le coefficient de la pluie est non significatif, mais on remarque que la p-value est faible $(13,7 \%)$ et amènerait en cas d'augmentation de notre seuil de tolérance à retenir une réduction de $0,83 \%([-1,931 ; 0,265])$. Le coefficient propre à un jour neigeux est par contre jugé significatif, avec une réduction de l'ordre de 4,1\% ([-6,8\%;-1,4\%]). Ces coefficients sont globalement conformes à ceux trouvés dans la littérature.

Les coefficients relatifs à la température moyenne, minimale ou maximale sur la journée ne sont pas significatifs, la température ne contribuerait donc pas a priori en semaine à une réduction du trafic moyen. Il en est de même concernant la vitesse minimum et maximum du vent sur la journée. Par contre, le coefficient propre à la vitesse moyenne du vent est significatif, mais conduit à une variation somme toute assez faible du volume de trafic journalier moyen: si la vitesse moyenne augmente de $1 \mathrm{mile} / \mathrm{h}$ (soit $1,6 \mathrm{~km} / \mathrm{h}$ ), le volume moyen journalier diminue de $0,16 \%$ ([-0,30 \% ;-0,025]). À titre de comparaison, Keay et Simmonds trouvaient une réduction de l'ordre de 0,23\% par miles/h supplémentaire. Les auteurs trouvaient par contre une influence de la température maximum et minimum statistiquement significative sur le débit journalier moyen. Néanmoins, les coefficients trouvés étaient très faibles (respectivement $+0,12 \%$ à chaque fois par degré supplémentaire).

\section{Autres modèles réalisés}

Un second modèle permet d'expliquer le trafic VLPL moyen journalier du week-end. Le coefficient de détermination est moins bon que le modèle précédent du vendredi, mais comparable à celui du modèle des premiers jours de semaine : $\mathrm{R}^{2}=61,3 \%$. Le modèle a donc un certain pouvoir explicatif.

Le week-end, l'utilisation du terme d'heures de pointe n'a plus vraiment de sens. L'utilisation de ces variables permet néanmoins de considérer l'impact des facteurs météorologiques sur les périodes horaires correspondantes de la journée (matin, mi-journée, fin de journée). Contrairement à ce qu'on aurait pu attendre, les coefficients relatifs aux variables de température et de vent ne sont pas significatifs. Il était par exemple envisageable de considérer qu'en cas d'augmentation des températures maximales le week-end, les trajets de loisirs augmenteraient et donc le volume journalier moyen aussi. Ce résultat s'explique peut-être par le fait que nous sommes en milieu interurbain, et que les trajets réalisés le week-end (hormis les retours des pistes de ski durant la période hivernale) sont plutôt des trajets longs et donc pas forcement de loisirs (aller voir des amis, faire des courses sur Lyon... Par contre, la neige et la pluie rentrent en compte dans la qualité de la conduite, et donc conditionnent la réalisation du déplacement, quel que soit son motif. Or le week-end les déplacements sont en général non contraints, donc a priori plus sensibles à ces facteurs météorologiques qui dégradent en général les conditions de conduite.

On constate ainsi que les nombres d'heures de pluie et de neige sont tous deux significatifs dans le modèle. De plus, les coefficients sont dans les deux cas plus forts que ceux trouvés en début de semaine ou le vendredi (dans le cas où ceux-ci sont significatifs). Cela confirmerait donc les éléments bibliographiques : l'influence de la pluie et de la neige serait plus importante le week-end qu'en semaine, et l'influence de la pluie serait toujours moins forte que celle de la neige.

L'ajout de la variable Occurrence_Pluie_24h entraîne une augmentation du $\mathrm{R}^{2}$ de $0,6 \%$, cette variable explique donc une petite partie de la variance du modèle. Chaque heure supplémentaire de la journée où la pluie est détectée entraîne une réduction de $0,72 \%([-1,5 \% ; 0,002 \%])$ du trafic moyen journalier du week-end. Si par exemple il pleut durant la moitié des heures de la journée, cela représente une réduction de $8,6 \%$. Pour rappel, les contradictions étaient fortes à ce sujet dans la littérature : en interurbain Hogema ne trouvait pas de réduction significative et Codling une réduction entre $7,6 \%$ et $17,8 \%$. En milieu urbain, Changnon relevait une réduction de $9 \%$ le week-end et Chung et al. de 7,9\% le samedi et 5,2\% le dimanche [24] [25] [26] [28].

L'ajout de la même variable, mais relativement à la neige, entraîne une augmentation du $\mathrm{R}^{2}$ plus importante de l'ordre de $1 \%$. Le coefficient est plus élevé que pour les autres jours étudiés : $-1,8 \%([-3,2 \% ;-0,37 \%])$ par heure où la neige est tombée sur la journée. En gardant à nouveau le critère de $12 \mathrm{~h}$, la réduction du volume moyen journalier du week-end avoisinerait les $22 \%$ ce qui est comparable aux valeurs trouvées en interurbain le week-end par Hanbali et Kuemmel (de $19 \%$ à $56 \%$ ), Hassan et Barker (15,3\%), Knapp et Smithson (entre $16 \%$ et $47 \%$ ) et Maze et al. (20\% en cas de bonne visibilité et de vent faible). Enfin, la significativité de la variable Occurrence_neige_HPM permet de conclure à une forte sensibilité de la demande le matin entre $7 \mathrm{~h}$ et 
$11 \mathrm{~h}$ par rapport aux autres périodes de la journée où les variables ne sont pas significatives ( $12 \mathrm{~h}-15 \mathrm{~h}$ et $16 \mathrm{~h}-20 \mathrm{~h}$ ). Le volume moyen journalier du week-end décroît en effet de $5,4 \%$ par heure où la neige est détectée sur ce créneau horaire. Précisons que l'intervalle de confiance à $95 \%$ est néanmoins large, de $-9,6 \% / \mathrm{h}$ à $-1,2 \% / \mathrm{h}$. Ce résultat pourrait signifier une prise de décision sur les activités de la journée le week-end en fonction des conditions météorologiques de la matinée. Comme on le voit sur les profils de débit journalier (même si les courbes peuvent être irrégulières en terme d'amplitude de la demande d'un samedi et d'un dimanche sur l'autre), la demande commence à augmenter en général vers $7 \mathrm{~h}$ et atteint un pic vers $12 \mathrm{~h}$ le samedi et le dimanche, ce qui pourrait corroborer cette hypothèse [9] [12] [27] [31].

Enfin, même si les coefficients des variables de pluie ou de neige journalières sont jugés non significatifs, les $\mathrm{p}$-values sont légèrement au-dessus du seuil de $5 \%$ (respectivement $5,7 \%$ et $7,0 \%$ ). On peut donc retenir ces valeurs avec un faible risque d'erreur. Les valeurs des réductions trouvées sont ainsi plus modestes (par rapport à l'application du critère de $12 \mathrm{~h}$ ) mais non négligeables puisque l'on relève une réduction de $5,1 \%$ pour la pluie et de $10,7 \%$ en cas de neige le week-end.

$\mathrm{Si}$ le coefficient relatif aux jours pluvieux est loin d'être significatif ( $p$-value de 45,3\%), une forte réduction de 18,7 .

\section{Conclusion et perspectives}

L'étude de la bibliographie à travers le prisme du modèle à quatre étapes montre que la structure des choix de déplacement peut être perturbée de différentes façons. Il est cependant difficile de savoir dans quelle mesure exactement chaque solution est mise en œuvre. De façon assez générale, les modifications dépendraient de plusieurs paramètres tels que la nature et l'intensité du phénomène météorologique en cause, le motif du déplacement, le type de milieu, la période temporelle, les caractéristiques socioculturelles de l'usager et sa localisation géographique. L'annulation du déplacement reste rare pour les trajets à fortes contraintes (déplacements professionnels, domicile-travail...) mais envisageable pour les trajets non-habituels (loisirs, achats...). La création de nouveaux déplacements n'est pas abordée, même si l'on peut concevoir que, dans certaines conditions météo (hautes températures par exemple), des déplacements de loisirs soient générés. Le choix de la destination est là aussi plutôt un problème propre aux trajets non-contraints, mais le thème reste peu étudié. Il est surprenant de constater qu'une partie des usagers semble envisager de changer d'itinéraire, alors qu'on peut supposer que l'ensemble des routes est affecté de façon identique par la météo, d'autant qu'en milieu urbain les déplacements sont souvent courts. Le transfert modal (en particulier depuis des modes « vulnérables » comme le vélo ou la marche) et le changement de l'horaire de départ sont les solutions $a$ priori les plus plébiscitées par les usagers, en particulier dans le cas des migrations pendulaires, car assez simples à mettre en place. Une certaine inertie des comportements est néanmoins dans tous les cas soulignée [16].

Nous nous sommes ensuite intéressés à l'ensemble des études utilisant les données des boucles de comptage véhiculaire, dans le but d'établir l'existence de réductions sur les volumes de trafic routier (qui peuvent être interprétés comme la demande) et de voir si ces réductions sont significatives ou non. Il n'est cependant pas possible de déterminer les causes de ces variations quand elles sont constatées. Les facteurs météorologiques semblant avoir le plus d'influence sur le trafic routier (et dont l'impact est reconnu par une grande majorité des auteurs) sont par ordre d'importance la neige puis la pluie. Les auteurs sont en tout cas rarement d'accord sur l'amplitude des réductions des différents facteurs météorologiques. Celles-ci dépendraient, assez logiquement là aussi, des facteurs évoqués précédemment pour la structure des choix de déplacement. On retiendra surtout que l'impact de la pluie sur les volumes routiers est considéré comme faible en semaine (entre $0 \%$ et $3 \%$ ) et comme assez variable et beaucoup plus fort le week-end (impacts sur les motifs non-contraints). L'impact de la neige est par contre important que ce soit en semaine ou le week-end, dans le même ordre d'importance que pour la pluie ( $5 \%$ à $15 \%$ en semaine contre $15 \%$ à $50 \%$ le week-end).

Cette étude bibliographique a ensuite été complétée par des analyses sur des données opérationnelles recueillies sur une section de l'autoroute A43. Des méthodes d'analyse statistique (superposition des intervalles de confiance, test de Mann-Whitney...) ont permis d'analyser divers échantillons de jours (secs, pluvieux, neigeux) et d'établir une influence de ces deux facteurs météorologiques sur le trafic de semaine de la section interurbaine étudiée et de confirmer une plus forte influence de la neige. Ces analyses statistiques ont ensuite été reproduites sur des données recueillies en milieu urbain sur la route $n^{\circ} 3$ du réseau de voies rapides de Tokyo et ont permis de confirmer ces analyses dans le cas des précipitations uniquement.

Des modèles de régression linéaire multiple permettant d'expliquer les volumes de trafic journaliers en fonction de différentes variables calendaires ont ensuite été réalisés. À ces modèles de base, nous avons ensuite ajouté un certain nombre de variables météorologiques (relatives à la pluie, à la neige, à la température ou au vent) afin de voir si ces dernières peuvent expliquer une partie de la variance 
du modèle. Selon les cas (modèle pour le lundi au jeudi, le vendredi, pour le week-end et un modèle pour le trafic poids lourd sur toute la semaine), des liens statistiquement significatifs avec le volume journalier moyen ont été trouvés. Les coefficients trouvés confirment en général les tendances dégagées par la littérature. On retiendra principalement une réduction moyenne du volume de trafic journalier moyen de $0,83 \%$ pour un jour pluvieux de début de semaine (lundi au jeudi) et de $5,1 \%$ le week-end, de $4,1 \%$ pour un jour neigeux de début de semaine et de $10,7 \%$ le week-end. Les modèles de trafic PL suggèrent une influence de la pluie négligeable sur la semaine, mais un effet fort de la neige car, même si ces trajets sont fortement contraints (critère économique), l'augmentation du risque d'accident en cas de neige est plus élevée que pour les conducteurs de VL. De plus, en cas de chute de neige des mesures d'interdiction de circulation destinées aux PL sont souvent mises en place. L'absence de données sur l'intensité des phénomènes météo et d'informations concernant l'état de la chaussée reste néanmoins un frein important à nos études.

Il n'est pas possible de mener les mêmes études sur la demande les vendredis, samedis et dimanches, notamment dans le cas particulier de la section Lyon-Chambéry, compte tenu des variations trop importantes de la demande. Des études de cas furent néanmoins menées afin de mettre en évidence les effets. Même si ces analyses de cas ne reposent sur aucun fondement statistique, elles permettent d'envisager un impact fort de la neige sur les trajets loisirs du week-end et du vendredi soir (de $20 \%$ à $40 \%$ de réduction par rapport au volume de trafic journalier moyen), et un impact assez variable de la pluie le vendredi (de $1 \%$ à $7 \%$ ). Pour ces cas, il semble nécessaire de se tourner plutôt vers une étude de l'influence des prévisions météorologiques plutôt que des conditions effectives (à l'aide de bulletins de prévisions, d'enquêtes auprès des usagers...).

\section{Références}

1. Prevedouros PD, Kongsil P (2003) Synthesis of the Effects of Wet Conditions on Highway Speed and Capacity. University of Hawaii-Manoa, Department of Civil Engineering, 19 p.

2. El Faouzi NE, Bouzebda S, Billot R (2010). Motorway travel time prediction based on toll data and weather effect integration. IET, Intelligent Transport Systems 4(4): 338-345.

3. Datla S, Sharma S (2007) Highway Traffic Volume Variations with Cold and Snows interactions. Paper prepared for presentation at the "Innovation in Traffic Operations and Management" Session of the 2007 Annual Conference of the transportation Association of Canada, $20 \mathrm{p}$.

4. Aaheim HA, Hauge KE (2005) Impacts of climate change on travel habits: a national assessment based on individual choices. In CICERO Report No. 2005:07. Center for International Climate and Environmental Research, Oslo, 38 p.
5. Sabir M, Koetse MJ, Rietveld P (2008) The impact of weather conditions on mode choice decisions: empirical evidence for the Netherlands. Forthcoming as Tinbergen Institute Discussion Paper. VU University, Amsterdam, $24 \mathrm{p}$.

6. Koetse MJ, Rietveld P (2009) The impact of climate change and weather on transport: an overview of empirical findings. In Transportation Research Part D 14, TRB, The National Research Council, Washington D.C., pp. 205-221.

7. Bonnel P (2004) Prévoir la demande de transport. Presses de l'Ecole Nationale des Ponts et Chaussées, 425 p.

8. Ortùzar JDD, Willumsen LG (2001) Modelling Transport. John Wiley \& Sons Inc, Chichester, 454p.

9. Maze TH, Agarwal M, Burchett G (2006) Whether Weather Matters to Traffic Demand, Traffic Safety, and Traffic Operations and Flow. In Transportation Research Record 1948, TRB, The National Research Council, Washington, D.C., pp. 170-176.

10. Koetse MJ, Rietveld P (2007) Climate change, Adverse Weather Conditions, and Transport: A Literature Survey. Study of the Vrije Universiteit, Department of Spatial Economics, Amsterdam, 23p.

11. Kilpeläinen M, Summala H (2007) Effects of weather and weather forecasts on driver behavior. Transportation Research Part F 10(4).

12. Hanbali R.M, Kuemmel D.A (1993) Traffic Volume Reductions Due to Winter Storm Conditions. Transportation Research Record 1387, TRB, National Research Council, Washington, D.C., 1993, pp. $159-164$.

13. Maki PJ (1998) Adverse Weather Traffic Signal Timing. ITE Annual Meeting, Institute of Transportation Engineers, Washington D. C., 8 p.

14. Hanson S, Hanson P (1977) Evaluating the impact of weather on bicycle use. In Transportation Research Record 629, TRB, National Research Council, Washington, D.C., pp. 43-48.

15. Richardson AJ (2000) Seasonal and weather impacts on urban cycling trips. TUTI Report 1-2000. The Urban Transport Institute, Victoria, Australia, 22p.

16. Khattak A, De Palma A (1997) The impact of adverse weather conditions on the propensity to change travel decisions: a survey of Brussels commuters. In Transportation Research Part A 31, TRB, National Research Council, Washington, D.C., pp. 181-203.

17. De Palma A, Rochat D (1999) Understanding individual Travel Decisions: Results from a Commuters Survey in Geneva. Transportation 26: 263-281.

18. Nankervis M (1999) The effect of weather and climate on bicycle commuting. Transportation Research Part A 33(6), pp. 417-431.

19. Bergström A, Magnusson R (2003) Potential for transferring car trips to bicycle during winter. In Transportation Research Part A 37, TRB, National Research Council, Washington, D.C., pp. 649-666.

20. Khattak A (1991) Driver response to unexpected travel conditions: effect of traffic information and other factors. PhD dissertation, Civil Engineering Department, Northwestern University, Evanston, Illinois.

21. Maze TH, Crum MR, Burchett G (2005) An Investigation of User Costs and Benefits of Winter Road Closures. Midwest Transportation Consortium, Iowa State University, pp. 40-60.

22. Rietveld R, Daniel V. (2004) Determinants of Bicycle use: Do municipal Policies Matter? Transportation Research, Part. A 38: 531-550.

23. Winters M, Friesen M, Koehoorn M, Teschke K (2007) Utilitarian Bicycling: A Multilevel Analysis of Climate and Personal Influences. American Journal of Preventive Medicine 32(1): $52-58$. 
24. Codling PJ (1974) Weather and road accidents. In J.A.Taylor (Ed.), Climatic Resources and Economic Activity. David and Charles Holdings, Newton Abbot, pp. 205-222.

25. Changnon SA (1996) Effects of summer precipitation on urban transportation. Climatic Change 32(4): 481-494.

26. Hogema JH (1996) Effects of rain on daily traffic volume and on traffic behavior. Report $n^{\circ}$ TM-96-B019, TNO Human Factors Research Institute, Netherlands, $14 \mathrm{p}$.

27. Hassan Y, Barker DJ (1999) The impact of unseasonable or extreme weather on traffic activity within Lothian Region, Scotland. Journal of Transport Geography 7: 209-213.

28. Chung E, Ohtani O, Kuwahara M (2005) Effect of Rainfall on Travel Time and Travel Demand. 5th ITS European Congress, $8 \mathrm{p}$.

29. Keay K, Simmonds I (2005) The association of rainfall and other weather variables with road traffic volume in Melbourne, Australia. Accident Analysis and Prevention 37: 109-124.

30. Fridstrøm L (1999) Econometric models of road use, accidents, and road investment decisions. TØI Report 457, Volume II, Chapter 4, Institute of Transport Economics, Oslo, pp. 89-91.
31. Knapp K, Smithson LD (2000) Winter storm event volume impact analysis using multiple-source archived monitoring data. In Transportation Research Record 1700, TRB, National Research Council, Washington, D.C., pp. 10-16.

32. Emmerson P, Ryley TJ, Davies DG (1998) The Impact of Weather on Cycle Flows. Transportation Research Labatory, Berkshire, England.

33. Goetzke F, Rave T (2006) Bicycle Use in Germany. Explaining Differences between Municipalities through Network Effects. Ph.D. dissertation, University of West-Virginia, Morgantown, USA.

34. Auffray B (2007) Impact of adverse weather on traffic conditions on an American highway, effect of the Sun Glare on Traffic Flow Quality. Travail de fin d'études d'ingénieur de l'ENTPE, Lyon, France, $60 \mathrm{p}$.

35. El Faouzi N.-E. (Ed.). Real-time monitoring, surveillance and control of road networks under adverse weather conditions. Effects of weather on traffic and pavement. State of the art and best practice report for COST Action TU0702, Les collections de l'INRETS, December 2010, R 283, ISSN 0768-9756, $146 \mathrm{p}$. 